\title{
Investigating the impact of the mycorrhizal inoculum on the resident fungal community and on plant growth
}

\author{
Maria M. Martignoni ${ }^{1}$, Jimmy Garnier ${ }^{2}$, Miranda M. Hart ${ }^{3}$, and Rebecca C. Tyson ${ }^{4}$ \\ ${ }^{1}$ Department of Mathematics, University of British Columbia, maria.martignonimseya@ubc.ca \\ ${ }^{2}$ Laboratoire de Mathématiques (LAMA), Université de Savoie, jimmy.garnier@univ-smb.fr \\ ${ }^{3}$ Department of Biology, University of British Columbia, miranda.hart@ubc.ca \\ ${ }^{4}$ Department of Mathematics, University of British Columbia, rebecca.tyson@ubc.ca
}

November 16, 2020

\begin{abstract}
In the last few decades, microbial inoculants have been used as organic fertilizers worldwide. Among the most widely used commercial products are arbuscular mycorrhizal (AM) fungi, as these fungi can associate with a variety of crops. Despite the potential benefits for soil quality and crop yield associated with AM fungal colonization, experiments assessing the persistence of the fungi in the field have yielded inconsistent results. Additionally, it is not yet clear whether or not the introduction of commercial inoculants could lead to changes to the resident fungal community, and eventually to invasion of the commercial products with a possible displacement of resident species. Here we use a partial differential equation model to assess the potential biodiversity risks and productivity benefits deriving from inoculation. We study the impact of AM fungal inoculation on the resident fungal community and on plant growth at a landscape scale. We determine how inoculant persistence and spread is affected by its competition with resident fungal species, by its mutualist quality, and by fungal dispersal. Our findings suggest that the increase in fungal abundance due to inoculation always leads to a short-term increase in host productivity, regardless of inoculant identity. However, the use of strongly competing inoculants constitutes a biodiversity risk, and may result in the invasion of low quality mutualists.
\end{abstract}

Keywords : Arbuscular mycorrhizal fungi (AMF), inoculum, inoculants, model, invasion, spread, persistence, establishment, agriculture, productivity, diversity, traveling waves, mutualism, competition 


\section{Introduction}

Arbuscular mycorrhizal (AM) fungi are beneficial fungi living in association with the roots of the vast majority of plants and facilitating plant access to nutrients in the soil (Smith and Read, 2010; Jeffries et al., 2003). Additional benefits provided to the plant by AM fungi are pathogen protection (Pozo and Azcón-Aguilar, 2007) and resistance against abiotic stress, such as drought or salinity (Latef et al., 2016). The plant, in exchange, provides the fungi with fixed carbon (Smith and Read, 2010). Because of their positive influence on plant fitness, AM fungi have been commercialised, and are widely used as organic fertilizers for a large variety of crops, such as maize, wheat, soybeans, tomatoes, or strawberries (Gianinazzi and Vosátka, 2004)).

Despite the potential benefits of field inoculation with commercial AM fungi, studies investigating the effective establishment of mycorrhizal inoculants have contradictory results (Berruti et al., 2016; Baum et al., 2015; Verbruggen et al., 2013). Some observations show field establishment and improved crop yield (Bender et al., 2019; Köhl et al., 2016; Buysens et al., 2016), while other studies show poor establishment of the inoculated species (Emam, 2016; Loján et al., 2017; Ryan and Graham, 2002). Moreover, it is still unclear how resident fungal communities respond to the introduction of a new fungal species (Thomsen and Hart, 2018; Hart et al., 2018). In some studies, inoculation caused little or no impact on the resident fungal community (Loján et al., 2017; Sỳkorová et al., 2012), while other observations show that resident species were displaced by the introduction of inoculants (Koch et al., 2011; Pellegrino et al., 2012; Symanczik et al., 2015; Bender et al., 2019). These last results in particular raise concerns over whether inoculated fungi could possibly spread to non-target areas causing a loss in soil biodiversity and, ultimately, negative effects on plant productivity (Schwartz et al., 2006; Hart et al., 2017; Ricciardi et al., 2017). It is therefore important to assess the conditions under which the use of commercial inoculants is beneficial or harmful, at short or large time scale.

Due to the challenges presented by large scale application and monitoring of inoculants, the current understanding of the impact of inoculation is largely based on short-term greenhouse experiments, comparing plant performance before and after inoculation (Berruti et al., 2016), while fewer studies have considered the long term persistence of inoculants in field conditions (Pellegrino et al., 2012; Farmer et al., 2007; Pellegrino et al., 2011; Sỳkorová et al., 2012; Kokkoris et al., 2019a). Additionally, less is known about the spread of non-pathogenic microbes in general (Litchman, 2010; Peay and Bruns, 2014; Peay et al., 2012; Davison et al., 2012; Thiet and Boerner, 2007). While some empirical work has been conducted on the dispersal of ectomycorrhizal fungi (Thiet and Boerner, 2007; Vellinga et al., 2009) no existing empirical or theoretical studies focus on the spread of AM inoculants in the field.

Mathematical modeling and computer simulations have proven to be useful tools for generating long-term predictions at a landscape scale when field trials are logistically not feasible(Jørgensen, 1994; Odenbaugh, 2005; Haller, 2014). Here we use a theoretical approach to determine the potential biodiversity risks and productivity benefits associated with inoculation. In particular, we aim to provide useful information for the selection of inoculants that are likely to promote plant growth while helping with the persistence or restoration of the resident fungal community. To undertake this task, we develop a partial differential equation model to investigate conditions for the establishment, persistence and spread of AM inoculants, and the resulting consequences on the resident fungal community and on plant growth.

Although many theoretical approaches have been used to study the spread of pathogenic fungi (Soubeyrand et al., 2008; Burie et al., 2008), the mechanisms driving the spread of AM fungi are substantially different, as these fungi are obligate symbionts, and their growth strictly depends on their mutualistic relationship with a host plant. Plant growth, in turn, is enhanced 
by fungal mutualists, where the degree of benefit depends on the specific fungal community composition and on the strength of the competitive interactions among the different fungal mutualists present (Smith and Read, 2010; Bever, 2002). Our model can be used to investigate how the spatio-temporal distribution of plant and fungal biomass is affected by mutualistic interactions between the plant and the fungi, and by competitive interactions between fungal species, what will give us information about the impact of the mycorrhizal inoculum on plant and fungal growth on a landscape scale. We will also investigate how the mutualistic quality of the inoculated fungus and its dispersal ability can affect the dynamics of the resident community. As our model is general, our results are not limited to a specific inoculant, nor to specific crops, but apply to any inoculants and crops.

\section{Model and Methods}

We consider the scenario in which an inoculant (whose biomass density is represented by the variable $m_{c}$ ) is introduced in a crop field inhabited by a resident community consisting of $N$ fungal species (with biomass density $m_{w j}$, where $j=1 . . N$ ). We assume that the plant is present across the landscape (with continuous biomass density $p$ ). With the term 'resident community' we indicate the assemblage of AM fungal species that were present before the introduction of the inoculant (not necessarily at equilibrium). With the term 'inoculant' we indicate a fungal species that is introduced to the resident community and can associate with the same plant as the resident fungi present.

AM fungi can spread in the soil through hyphae (Friese and Allen, 1991), or through short or long range dispersal of spores by different agents (Warner et al., 1987). To model fungal spread, we will consider only short range dispersal of fungal biomass, either through hyphae or spores (not distinguishable in the model). We assume therefore that fungi disperse by random diffusion, and quantify the dispersal ability of each fungal species by its diffusion coefficient ( $D_{c}$ for the inoculant and $D_{w j}$ for each of the resident fungal species).

All fungi are in a mutualistic relationship with the plants, where nutrients exchanged are phosphorus (fungi to plant) and carbon (plant to fungi). Competition between fungal species can reduce the amount of carbon received by both competitors. Mathematically, we write

$$
\begin{aligned}
& \partial_{t} p(x, t)=f_{p}\left(p, m_{c}, m_{w_{j}}\right), \\
& \partial_{t} m_{c}(x, t)=D_{c} \partial_{x}^{2} m_{c}+f_{m_{c}}\left(p, m_{c}, m_{w_{j}}\right), \\
& \partial_{t} m_{w_{j}}(x, t)=D_{w_{j}} \partial_{x}^{2} m_{w j}+f_{m_{w_{j}}}\left(p, m_{c}, m_{w_{j}}\right), \quad \text { with } j=1 . . N,
\end{aligned}
$$

where the growth functions $f_{p}, f_{m_{c}}$ and $f_{m_{w j}}$ describe the interactions between the plant and the AM fungi and were introduced in Martignoni et al. (2020b). More precisely, the form of the growing functions is the following:

$$
\begin{aligned}
& f_{p}=\overbrace{\alpha_{c} f_{h p}\left(p, m_{c}\right)+\sum_{j=1}^{N} \alpha_{w_{j}} f_{h p}\left(p, m_{w_{j}}\right)}^{\begin{array}{c}
\text { phosphorus } \\
\text { received }
\end{array}}-\overbrace{\beta_{c} f_{c p}\left(p, m_{c}\right) C_{m_{c}}-\sum_{j=1}^{N} \beta_{w_{j}} f_{c p}\left(p, m_{w_{j}}\right) C_{m_{w_{j}}}+\overbrace{r_{p}(p)}^{\text {plant }}}^{\begin{array}{c}
\text { carbon } \\
\text { supplied }
\end{array}}, \\
& f_{m_{c}}=\beta_{c} f_{c m}\left(p, m_{c}\right) \quad C_{m_{c}} \quad-\alpha_{c} f_{h m}\left(p, m_{c}\right)-\mu_{m_{c}} m_{c}^{2},
\end{aligned}
$$

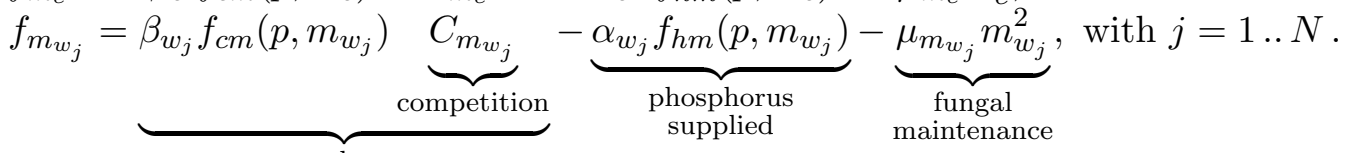

$$
\begin{aligned}
& \begin{array}{l}
\text { carbon } \\
\text { received }
\end{array}
\end{aligned}
$$


A description of the model parameters is given in Table 1. Below we explain the key components of the model. The complete version of the model is provided in the supplementary information, Eq. (6).

Nutrient exchange with the plant is determined by the functions $f_{c p}$ and $f_{c m}$ (carbon transfer), and $f_{h p}$ and $f_{h m}$ (phosphorus transfer). Carbon transfer depends linearly on plant and fungal biomass densities (i.e., $\propto p m_{s}$, where $s=w_{j}, c$ ), while phosphorus transfer depends on fungal biomass density only, when the plant is large enough, while plant biomass limits phosphorus transfer at low density (i.e., $\propto \frac{p}{d+p} m_{s}$, where $\left.s=w_{j}, c\right)$ (Martignoni et al., 2020a). Fungal species may differ in their ability to transfer phosphorus to the plant ( $\alpha_{s}$ parameters) and in their access to plant carbon ( $\beta_{s}$ parameters), where the $\alpha_{s} / \beta_{s}$ ratio will be used throughout the manuscript to characterise the mutualist quality of a fungal species. Plant growth in the absence of the fungi is logistic, and expressed by the function $r_{p}(p)$. AM fungi can not survive in the absence of the host plant, therefore no intrinsic growth terms appear in $f_{m_{w_{j}}}$ and $f_{m_{c}}$.

The functions $C_{m_{c}}$ and $C_{m_{w_{j}}}$ quantify the reduction in access to host carbon by fungal species, either the inoculant $\left(m_{c}\right)$ or a resident fungal species $\left(m_{w_{j}}\right)$, due to competition with other fungi in the community. The competitive effect exerted by a fungal species on another depends on its competitive ability $\left(a_{s_{1}, s_{2}}\right.$-parameters) and on the proportion, in terms of biomass, that this species occupies within the competing community. We write

$$
\begin{aligned}
C_{m_{c}} & =\frac{\sum_{j} a_{w_{j} c} m_{w_{j}}}{\sum_{j} a_{w_{j} c} m_{w_{j}}+\left(\sum_{j} m_{w_{j}}\right)^{2}}, \\
C_{m_{w_{j}}} & =\frac{a_{c w_{j}} m_{c}+\sum_{i \neq j} a_{w_{i} w_{j}} m_{w_{i}}}{a_{c w_{j}} m_{c}+\sum_{i \neq j} a_{w_{i} w_{j}} m_{w_{i}}+\left(m_{c}+\sum_{i \neq j} m_{w_{j}}\right)^{2}},
\end{aligned}
$$

where large $a_{s_{1}, s_{2}}$ parameters result in weak competition, and small $a_{s_{1}, s_{2}}$ parameters result in strong competition, as the carbon uptake capacity of a species is significantly reduced for small $a_{s_{1}, s_{2}}$ but not for large $a_{s_{1}, s_{2}}$ (i.e., the terms $C_{m_{c}}$ and $C_{m_{w_{j}}}$ tend to 1 for large $a_{s_{1}, s_{2}}$ and tend to zero for small $a_{s_{1}, s_{2}}$ ). Note that when only one species is present, Eq. (3) tends to 1 as no competition is observed. To ensure coexistence of resident species, we assume $a_{w_{j} w_{i}}$ parameters to be large (i.e., competition between resident fungal species is small) (Martignoni et al., 2020b), while we vary parameters $a_{c w_{j}}$ and $a_{w_{j} c}$, quantifying the competitive interactions between the inoculant and resident fungal species.

We will first study the model dynamics analytically (see section 2.1) to quantify the fungal dispersal patterns that can be observed, with respect to the entire parameter space, and identify which factors can accelerate the invasion process or prevent an invasion. Through numerical simulations, we will then use the model to provide useful practical insights into agroecosystem management (see section 2.2), e.g., evaluating the impact of inoculation on productivity and assessing the risk of dispersal of low quality mutualists between neighbouring fields. Numerical simulations will be computed in Matlab R2017a using a semi-implicit scheme (Tyson et al., 2000), where the reaction term is solved explicitly and the diffusion term is solved implicitly. The discretization used for the space is $\mathrm{d} x=0.05$, and the discretion used for the time is $\mathrm{d} t=0.05$.

\subsection{Investigating inoculum persistence and spread}

To simplify the analysis, we assume that resident fungal species have the same competitive effect on each other (i.e., $a_{w_{i} w_{j}}=a_{w w}$ for all $i, j$ ), that each of the resident fungal species present has the same competitive effect on the inoculated species (i.e, $a_{w_{j} c}=a_{w c}$ for all $j$ ), and that the inoculant has the same competitive effect on each resident fungal species (i.e., 
$a_{c w_{j}}=a_{c w}$ for all $j$ ). Additionally, we assume that resident fungal species have identical fungal parameters, while resident and inoculated fungal species differ only in their diffusive ability ( $D_{c}$ and $D_{w}$ parameters), and mutualist quality (i.e., $\alpha_{s}$ and $\beta_{s}$ parameters).

We investigate four different scenarios which differ by the intensity of competition between the inoculum and resident fungal species, as described in Table 2 . These scenarios are representative for the whole parameter space for which mutualism establishment can occur (Martignoni et al., 2020a,b). In case (A) competition between resident fungal species and the inoculated species is weak (i.e., parameters $a_{w c}$ and $a_{c w}$ are large), e.g., due to functional complementarity between the inoculant and the resident species present. In cases (B) and (C) competition between resident fungal species and the inoculated species is asymmetric, where the superior competitor is either the resident fungal species (if $a_{w c}<a_{c w}$ ) or the inoculated species (if $a_{c w}<a_{w c}$ ). In case (D) competition between resident fungal species and the inoculated species is strong ( $a_{c w}$ and $a_{w c}$ are small), e.g., due to functional overlap between the inoculant and any of the resident species present. Quantitative criteria and steady stability for these four cases in the non-spatial case are given in Table 2. Since the output of case (D) depends on the initial distribution of inoculant, we distinguish between case (Da), where the inoculant is introduced over a small proportion of the field, and case (Db), where the inoculant is introduced over a large proportion of the field.

Analytical criteria to determine a lower bound for the spreading speed of resident fungi and of the inoculant will be provided for cases (A)-(C), while the sign of the spreading speed will be determined for cases (Da). Analytical spreading speed will be compared with numerical solutions. Case $(\mathrm{Db})$ will be investigated numerically.

\subsection{Agroecosystem management issues}

To investigating the spread of inoculants between neighboring fields, we consider a landscape consisting of two fields next to each other. We assume that the field on the left comprises only the fungal inoculant, while the field on the right is inhabited by an established resident fungal community. We use numerical simulations to look at the spread of the inoculant from the left to the right field, when competition between the inoculant and resident fungal species is weak or strong. As it has been proposed that commercial inoculants invest more in their own reproduction at the expenses of their mutualistic relationship with the plant (Kokkoris et al., $2019 \mathrm{~b}$ ), we assume that the inoculant has lower resource exchange capacity than resident species (i.e., lower $\alpha_{c} / \beta_{c}$ ratio). We consider the situation in which the resident community consists of two fungal species, and the situation in which only one resident fungal species is present.

Finally, to evaluate productivity in response to inoculation, we assume that inoculation occurs at the left edge of the landscape and we simulate plant and fungal growth and spread over time, when the inoculant can be a weak or a strong competitor. We focus on the effect of inoculation in depleted soils (i.e., when density of resident fungi is low), as the model predicts that the introduction of inoculants has little or no effect on plant growth in the presence of a well-established resident fungal community (Martignoni et al., 2020b).

\section{Results}

\subsection{Investigating inoculant persistence and spread}

When varying the strength of competition between the community and the inoculated species we encounter four possible combinations (cases (A)-(D)), summarised in Table 2. Case (D) 
can lead to two possible outcomes depending on the initial distribution of inoculants, cases (Da) and (Db), shown in Fig. 1. These scenarios cover the whole parameter space for which mutualism establishment can occur (Martignoni et al., 2020a,b), and quantitative bounds on the parameters corresponding to each of the cases discussed are given in Table 2 . We find that the inoculant can either not persist in the soil (cases (B) and (Da)), coexist with the resident fungal community (case (A)), or competitively exclude resident fungal species (cases (C) and $(\mathrm{Db}))$. A complete mathematical analysis is presented in the supplementary information. Below we discuss the key results and ecological insights emerging from the analysis.

The inoculant does not persist (cases (B) and (Da)): If resident fungi are strong (initially localised) competitors, the community prevents the persistence of the inoculant, regardless of whether the inoculant is a weak (case (B)) or strong competitor (case (Da)). In case (B), a weakly competing inoculant is displaced by resident fungal species at a speed with lower bound given by

$$
c_{w}=2 \sqrt{D_{w} p_{c}^{*}\left(\frac{q_{c m} \beta_{w} a_{c w}}{a_{c w}+m_{c}^{*}}-\frac{q_{h m} \alpha_{w}}{p_{c}^{*}+d}\right)},
$$

where $D_{w}$ is the dispersal ability of resident fungal species, $\alpha_{w}$ and $\beta_{w}$ are respectively the phosphorus and carbon exchange abilities of resident fungal species, $a_{c w}$ indicates the competitive effect of the inoculant toward resident fungal species, and $q_{c m}, q_{h m}$, and $d$ are other model parameters defined in Table 1 . Parameters $m_{c}^{*}$ and $p_{c}^{*}$ are respectively the biomass density of the inoculant and plant at equilibrium in the absence of resident fungi. From the restrictions imposed on the parameters in cases (B) and (D) (see Table 2), we know that $c_{w}^{*}>0$. From the analytical expression in Eq. (4) and from the numerical simulations shown in Fig. 2 (case (B)) we can see that resident fungal species displace the inoculant at a speed that increases with increasing mutualist quality of the inoculant (as higher values of $p_{c}^{*}$ and $m_{c}^{*}$ are observed when the $\alpha_{c} / \beta_{c}$ ratio is high) and with decreasing competitive ability of the inoculant (i.e., with larger $a_{c w}$ ). In contrast, the displacement speed is independent of the dispersal ability of the inoculant and on the number of resident fungal species in the community (i.e., $D_{c}$ and $N$ do not appear in Eq. (4)).

For a combination of low resident fungal density and high (initially localised) propagule pressure (case (Da)), the inoculant can temporarily establish and spread (see Fig. 1, case $(\mathrm{Da}), \mathrm{T}=0,20)$. The initial spread of the inoculant accelerates plant growth, but prevents the growth of resident fungi in areas where inoculant density is high. Beyond the dispersal range of the inoculant, the density of resident fungi increases and so does plant productivity, eventually reaching a higher density than in the region where only the inoculant is present (Fig. 1, case (Da), $\mathrm{T}=30,40)$. A higher plant biomass density determines higher resource availability, and gives a competitive advantage to resident fungal species with respect to the inoculant. Eventually, the resident community is able to stop the spread of the inoculant and recolonize the field (see Fig. 1, case (Da), $\mathrm{T}=60,100$ ). Numerical simulations show that when recolonization is possible, resident fungal species displace the inoculant at a speed that increases with increasing mutualist quality, and with increasing diversity of resident fungal species, but that decreases with increasing dispersal ability of the inoculant (see Fig. 2, case (Da)).

Inoculant and resident community coexistence (case (A)): When competition between the inoculant and the existing community is low (e.g., the inoculated species is functionally complementary to resident fungal species), coexistence is possible and the inoculant can establish and spread in the field. In this case, inoculation has a positive effect on plant growth, due to an increase in diversity in the fungal community and therefore to a better use 
of the resource available (i.e., plant carbon) (Martignoni et al., 2020b). On the other hand, the persistence of the inoculant leads to a reduction in the total density of resident fungi. This reduction, however, does not threaten community survival.

The spreading speed of the inoculant $c_{c}$ can be expressed as

$$
c_{c}=2 \sqrt{D_{c} p_{w}^{*}\left(\frac{q_{c m} \beta_{c} a_{w c}}{a_{w c}+N m_{w j}^{*}}-\frac{q_{h m} \alpha_{c}}{p_{w}^{*}+d}\right)},
$$

where $D_{c}$ is the dispersal ability of the inoculant, $\alpha_{c}$ and $\beta_{c}$ are respectively the phosphorus and carbon exchange abilities of the inoculant, $a_{w c}$ is a parameter indicating the competition strength of the resident fungal species towards the inoculant, and $q_{\mathrm{cm}}, q_{\mathrm{hm}}$, and $d$ are other model parameters defined in Table 1. Parameters $p_{w}^{*}$ and $N m_{w j}^{*}$ are respectively the plant biomass density and total biomass density of resident fungal species at equilibrium in the absence of the inoculant. From the restrictions imposed on the parameters (see Table 2), we know that $c_{c}^{*}>0$. From the analytical expression in Eq. (5) and from numerical simulations shown in Fig. 2 (case (A)) we can see that the spreading speed of the inoculant increases with increasing dispersal ability and with decreasing mutualist quality of the inoculant (i.e., for small $\alpha_{c} / \beta_{c}$ ). Additionally, the spreading speed is lower in the presence of a diverse resident community (i.e., for high $N$ ).

\section{Invasion of the inoculant and displacement of the resident community (cases}

(C) and (Db)): If the inoculant is competitively superior to the resident fungal species, the inoculant will establish, displace resident fungi and spread, causing regional biodiversity loss (case (C)). Diversity of resident fungal species increases community resilience to invasion (Martignoni et al., 2020b). That is, the introduction of a strongly competing inoculant leads to invasion of the inoculant if diversity in the resident community is low, but constitutes a lower risk if diversity in the resident community is high. Additionally, inoculants with low mutualist quality (i.e., low $\alpha_{c} / \beta_{c}$ ) are most likely to invade (Martignoni et al., 2020b), as the quantitative criteria of case (C) provided in Table 2 are more likely to be satisfied for small $\alpha_{c} / \beta_{c}$ ratio. Abundant inoculation over a large proportion of field, combined with low abundance of resident fungi, can also lead to invasion (Fig. 1, case (Db)).

The analytical expression for the speed of invasion of the inoculant in case $(C)$ is equivalent to the speed derived for case (A) (see Eq. (5)), despite the fact that case (A) implies coexistence of the inoculant with resident fungal species, while in case (C) resident fungal species are displaced by the inoculant. Diversity in the resident community contributes to a reduction of the invasion speed, while low mutualist quality and high dispersal ability of the inoculant contribute to an increase (see Fig. 2, case (C)).

\subsection{Agroecosystem management issues}

Neighboring fields: The spread of a weakly competing inoculant in a field causes a decline in the biomass density of resident fungi (without, however, causing the extinction of resident species), and an increase in plant biomass density (case (A), discussed above). The decline in resident fungi and the increase in plant productivity are more pronounced when diversity in the resident fungal community is low (see Fig. 3). A strongly competing inoculant can not spread into a field inhabited by two or more strongly competing resident species (Fig. 3(a)). When only one resident fungal species is present however, a strongly competing inoculant with low mutualist quality is able to invade the field, displace the resident fungi and cause a reduction in plant productivity (see Fig. 3(b)). 
Inoculation and productivity: Generally, an increase in fungal abundance automatically corresponds to an increase in plant growth rate, independent of the identity of the inoculant and its persistence in the long term (see the first two subfigures of Fig. 1, case (Da) and Fig. 4). In Fig. 4 we simulate plant and fungal growth over a landscape, following inoculation with a weakly competing inoculant in a community consisting of strongly competing resident fungi (case (B)). Despite the fact that the inoculant does not persist in the field (see Fig. $4, \mathrm{~T}=40$ ), inoculation significantly enhances the plant growth rate and the growth rate of resident fungal species (Fig. $4, \mathrm{~T}=20,40$ ). Inoculation with strongly competing species can also lead to an initial enhancement in plant growth (see the first two subfigures of Fig. 1, case (Da)), but subsequently lead to a displacement of resident fungi, and to negative long term consequences for plant productivity (see case (C), Table 2).

\section{Discussion}

\subsection{Investigating inoculum persistence and spread}

The inoculant does not persist (cases (B) and (Da)): We found that if resident fungal species compete strongly with the inoculant (e.g., if no niche space is available in the soil), persistence of the inoculant will not occur. Experiments have reported that competition can have a strong effect on inoculants establishment (Bender et al., 2019; Niwa et al., 2018; Thomsen and Hart, 2018), where high species richness in the resident community may act as a competitive advantage through a better exploitation of the resources available (Mallon et al., 2015). In the model, the displacement of the inoculant by resident fungi may occur through two mechanisms: either resident fungi are superior competitors, and displace the weakly competing inoculant through direct competition between fungi for resources (case (B)), or through an improved mutualistic relationship with the plant (case (Da)). Indeed, in case (Da), high diversity in the resident fungal species allows for maximum resource exchange with the plant. Increased resource exchange contributes to higher plant density and thus higher resource availability (i.e., plant carbon), constituting an indirect competitive advantage of resident fungi with respect to the inoculant, once plant density has increased sufficiently.

The model predicts that, when the biomass density of the resident community is low, high propagule pressure can lead to the local establishment of the inoculant even if resident species are strong competitors (Verbruggen et al., 2013; Streeter, 1994; Bender et al., 2019; Niwa et al., 2018; Sỳkorová et al., 2012). However, we found that if the inoculant is introduced only over a small proportion of field, persistence may not occur, as resident fungi will eventually outcompete the inoculant and recolonize the field (see Fig. 1, case (Da)).

Inoculant and resident community coexistence (case (A)): Our results suggest that if competition between the introduced inoculant and resident fungal species is weak, e.g., due to small niche overlap between resident fungi present and the inoculated species, the inoculant will coexist with the rest of the community (case (A), Table 2). For example, if the introduced species comes to occupy an empty niche space available in the soil (Herbold and Moyle, 1986; Lekevičius, 2009; MacArthur and Levins, 1967), the inoculant will establish, persist, and spread in the field. Experiments agree with these predictions, showing that inoculum establishment in the presence of a resident fungal community is possible, and niche availability is one of the factors determining inoculation success (Verbruggen et al., 2013; del Mar Alguacil et al., 2011; Sỳkorová et al., 2012; Farmer et al., 2007; Mummey et al., 2009). Mummey et al. (2009) showed that the identity of the fungal species present before inoculation is a strong determinant of inoculant establishment, and Farmer et al. (2007) found 
that establishment was more successful when the inoculant had a functionality different from that of the indigeneous fungal species present in the field.

Invasion of the inoculant and displacement of the resident community (cases (C) and (Db)): We find that highly competitive fungi are likely to become invaders and cause a biodiversity loss in the resident fungal community. Concerns about the invasiveness of commercial inoculants and the possible detrimental consequences on ecosystem functionality have been raised by the scientific community (Schwartz et al., 2006; Hart et al., 2017; Ricciardi et al., 2017; Thomsen and Hart, 2018), and a decline in resident AM fungi following inoculation success has been experimentally observed (Koch et al., 2011; Pellegrino et al., 2012; Symanczik et al., 2015; Bender et al., 2019). Commercial fungi are subjected to intense breeding pressure (Gianinazzi and Vosátka, 2004), which leads to the selection of highly competitive traits such high sporulation and a propensity to invest in their own reproduction rather than in the mutualistic relationship with the plant (Kokkoris et al., 2019b; Calvet et al., 2013; Jin et al., 2013). Our analysis indicates that there is a risk of invasion from inoculants, and that competitive inoculants with lower mutualist quality are more likely to become invasive.

The presence of a diverse resident community can mitigate the invasion risk by reducing the invasion speed (see Eq. (5)), or by making establishment of the invader less likely (see Fig. 3). Additionally, when resident species abundance is low, the introduction of strongly competing inoculants can result in invasion if inoculants are introduced over a large portion of field (Fig. 1, case $(\mathrm{Db})$ ). Hence, our results underscore the presence of an increased invasion risk when competitive inoculants are used in conjunction with agricultural management practices that reduce the abundance and diversity of resident fungi, such as tillage, chemical fertilization strategies, or fallow periods (Karasawa and Takebe, 2012; Schnoor et al., 2011; Curaqueo et al., 2011; Säle et al., 2015).

\subsection{Speed of invasion}

We obtain concrete analytical criteria for the speed of invasion of inoculants in a field inhabited by a resident fungal community. The replacement of resident species by an invasive species has already been investigated using Lotka-Volterra competition models (Hosono, 1998; Okubo et al., 1989). However, an analytical expression for the spreading speed has been obtained only recently (Holzer and Scheel, 2012; Girardin, 2017), and only in a homogeneous environment. Other studies have described the spread of competitors in spatially heterogeneous (Goldstein et al., 2019; Real and Biek, 2007) or periodic environments (Kinezaki et al., 2003). In our model the resource (i.e., the plant) is involved in a mutualistc interaction with the species in competition (i.e., the fungi), and so the local plant density depends on the local fungal community composition and density. Thus, since we are investigating invasion scenarios where the fungi are not uniformly distributed through space, the environment for the competitors is heterogeneous, and the heterogeneity evolves with the spread of the competing species. In this heterogeneous context, we obtain analytical expressions for the speed of invasion of competitors (i.e., resident and inoculated fungal species).

Previous studies investigating traveling wave solutions in competitive systems have found that the speed of propagation can be linear or non-linear (Okubo et al., 1989; Lewis et al., 2002; Huang, 2010; Girardin, 2017), depending for example on interspecific competition (Roques et al., 2015; Huang and Han, 2011), or on the dispersal ability of the invader (Holzer and Scheel, 2012). Our analysis shows that the linear speed appears to be a valid approximation in two of the monostable cases (Fig. 2, cases (A) and (C)). However, for high mutualist quality and high competitive ability of the inoculant (Fig. 2, case (B)), the speed appears to be 
non-linear. Similarly, Roques et al. (2015) found non-linearity when interspecific competition is high, and linearity when interspecific competition is low.

In the bistable case, we are able to show that a strong competitor is displaced by a resident community consisting of two or more species that compete weakly between each other, but we are unable to obtain an analytic expression for the speed of spread. Guo et al. (2019) also investigated a bistable system and found that two weaker competitors can displace a stronger competitor, under certain parametric conditions. As the competition system studied by Guo et al. (2019) is similar to the one discussed here, we could expect to gain more analytical insights into the bistable case by adapting the Guo et al. (2019) analysis to our specific case. This task, however, is beyond the scope of the current paper, and is left to future work.

\subsection{Agroecosystem management issues}

Competitiveness has been seen as a desirable trait in fungal inoculants, as it increases establishment success (Verbruggen et al., 2013; Farmer et al., 2007). However, we find that the spread of competitive and less mutualistic inoculants leads to the displacement of the resident fungi and a decrease in plant productivity (Koch et al., 2011; Pellegrino et al., 2012; Symanczik et al., 2015; Bender et al., 2019). Invasion is more likely to happen if strongly competing inoculants are abundandly distributed over a large proportion of the field.

We show that the use of weakly competing inoculants is desirable, as their spread can boost plant growth and the growth of resident fungi, even when inoculants do not persist in the field. This result is in agreement with the observations of del Mar Alguacil et al. (2011). Therefore, we suggest that weakly competing inoculants are a better choice in agricultural contexts, as strongly competing fungi may lead to reduced soil biodiversity and, ultimately, reduce ecosystem functioning.

We show that inoculant abundance, and not necessarily the type of inoculant itself, can contribute to a short-term increase in plant productivity in depleted soils (i.e., when fungal biomass is low) (see the first two subfigures of Fig. 1, case (Da) and Fig. 4), while the increase is less significant when fungal abundance in the resident community is high (Martignoni et al., 2020b). Experiments investigating variation in crop yield following field inoculation with AM fungi agree with these results, by showing that the immediate consequences of inoculation can range from positive (Zhang et al., 2019; Ceballos et al., 2013; Hijri, 2016; Omirou et al., 2016) to no significant effect (Hamel and Smith, 1991; Loján et al., 2017; Ryan and Graham, 2002; Emam, 2016). Our observations support the view that short term positive impact on plant growth can be related to an increase in fungal abundance, rather than to fungal identity (Wagg et al., 2015; Gosling et al., 2016; Lekberg and Koide, 2005; Pellegrino et al., 2011; del Mar Alguacil et al., 2011).

\section{Conclusion}

We show that the use of weakly competing inoculants can lead to improved crop yield and soil quality, but the use of strongly competing fungi constitutes a biodiversity risk. Our model provides concrete criteria to assess the risks and benefits of inoculation and to determine the spreading speed of inoculants in the field. We show that invasion of the inoculated species, and the subsequent displacement of resident fungi, is unlikely to occur in the presence of an established resident community. However, the invasion risk increases if strongly competing inoculants with low mutualistic quality are introduced in fields presenting low abundance and diversity of resident fungi.

Our framework shows that monitoring plant growth is not sufficient to properly evaluate 
the long-term impact of inoculation, as the presence of a resident fungal community plays a critical role in determining inoculum establishment success and productivity. To confirm our hypotheses, there is an urgent need for empirical studies considering the effect of inoculation on the resident community over space and time. We hope that the theoretical findings exposed in this manuscript will help to focus future experimental efforts in productive directions. Our model is based on the consumer-resource framework of mutualism (Holland and DeAngelis, 2010), where growth rates can be directly associated with nutrient exchange, making an interplay between theory and experiments feasible. Indeed important model parameters (such as $\alpha$, the fungal ability to transfer phosphorus to the plant) can be estimated by monitoring phosphorus or carbon transfer between the plant and the fungus over time.

Finally, we also see further benefits of our work outside the context of mycorrhizal inoculants. As our model is general, the framework presented here can be used to improve our understanding of mechanisms behind the invasion of other symbiotic fungi (Dickie et al., 2016; Litchman, 2010), or animal pollinators (Acosta et al., 2016).

\section{Acknowledgment}

JG acknowledges NONLOCAL project (ANR-14-CE25-0013), GLOBNETS project (ANR-16CE02-0009) and the European Research Council (ERC) under the European Unions Horizon 2020 research and innovation program (grant agreement No 639638, MesoProbio). MMH acknowledges NSERC Discovery Grant RGPIN-2018-237774. RCT acknowledges NSERC Discovery Grant RGPIN-2016-05277 and the 'Make our planet great again (MOPGA)' grant.

Table 1: Description of variables and parameters of the model.

\begin{tabular}{||l|l||}
\hline Symbol & Description \\
\hline$p$ & Plant biomass density \\
$m_{w_{j}}$ & Biomass density of resident fungal species \\
$m_{c}$ & Biomass density of fungal inoculant \\
$D_{w_{j}}$ and $D_{c}$ & Fungal dispersal ability \\
$\alpha_{w_{j}}$ and $\alpha_{c}$ & Fungal ability to transfer phosphorus to the plant \\
$\beta_{w_{j}}$ and $\beta_{c}$ & Fungal ability to uptake carbon from the plant \\
$a_{w_{j} c}$ & Competitive effect of $m_{w_{j}}$ toward $m_{c}$ \\
$a_{c w_{j}}$ & Competitive effect of $m_{c}$ toward $m_{w_{j}}$ \\
$a_{w_{i} w_{j}}$ & Competitive effect of $m_{w_{i}}$ on $m_{w_{j}}$ \\
$\mu_{m_{w_{j}}}$ and $\mu_{m_{c}}$ & Fungal maintenance rates \\
$\mu_{p}$ & Plant maintenance rate \\
$d$ & Half-saturation constant \\
$q_{h m}$ & Conversion factor (phosphorus to fungal biomass density) \\
$q_{c m}$ & Conversion factor (carbon to fungal biomass density) \\
$q_{h p}$ & Conversion factor (phosphorus to plant biomass density) \\
$q_{c p}$ & Conversion factor (carbon to plant biomass density) \\
\hline Note: $m_{c}^{*}, m_{w}^{*}$ and $p^{*}$ represent the values of fungal and plant biomass at equilibrium. \\
\hline
\end{tabular}




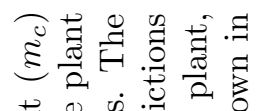

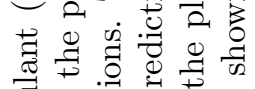

ज्ञ $\overrightarrow{0}$ :

ज $\overrightarrow{00}$.

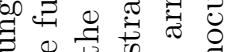

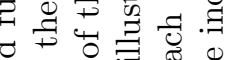

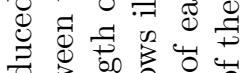

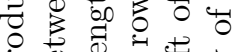

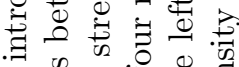

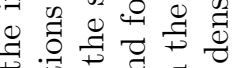

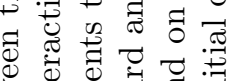

递卷寄

- 额

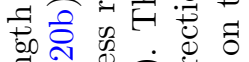

จำ

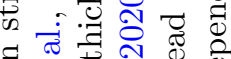

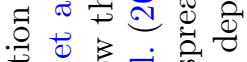

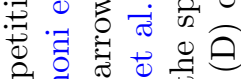

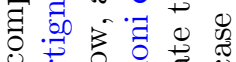

讨

월 웅

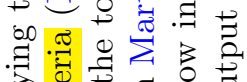

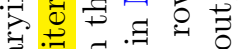

ปี

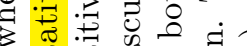

20.0 .

寻

\& 80.

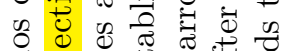

0.00

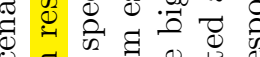

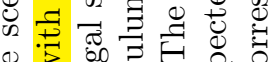

उ 3 .

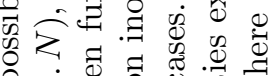

1 :

है $\|$ :

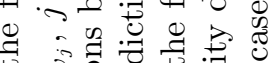

हैं 윰

进

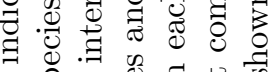

की

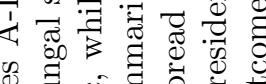

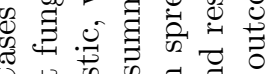

ช

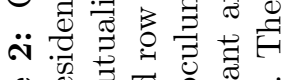

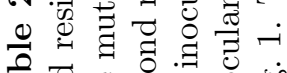
๘

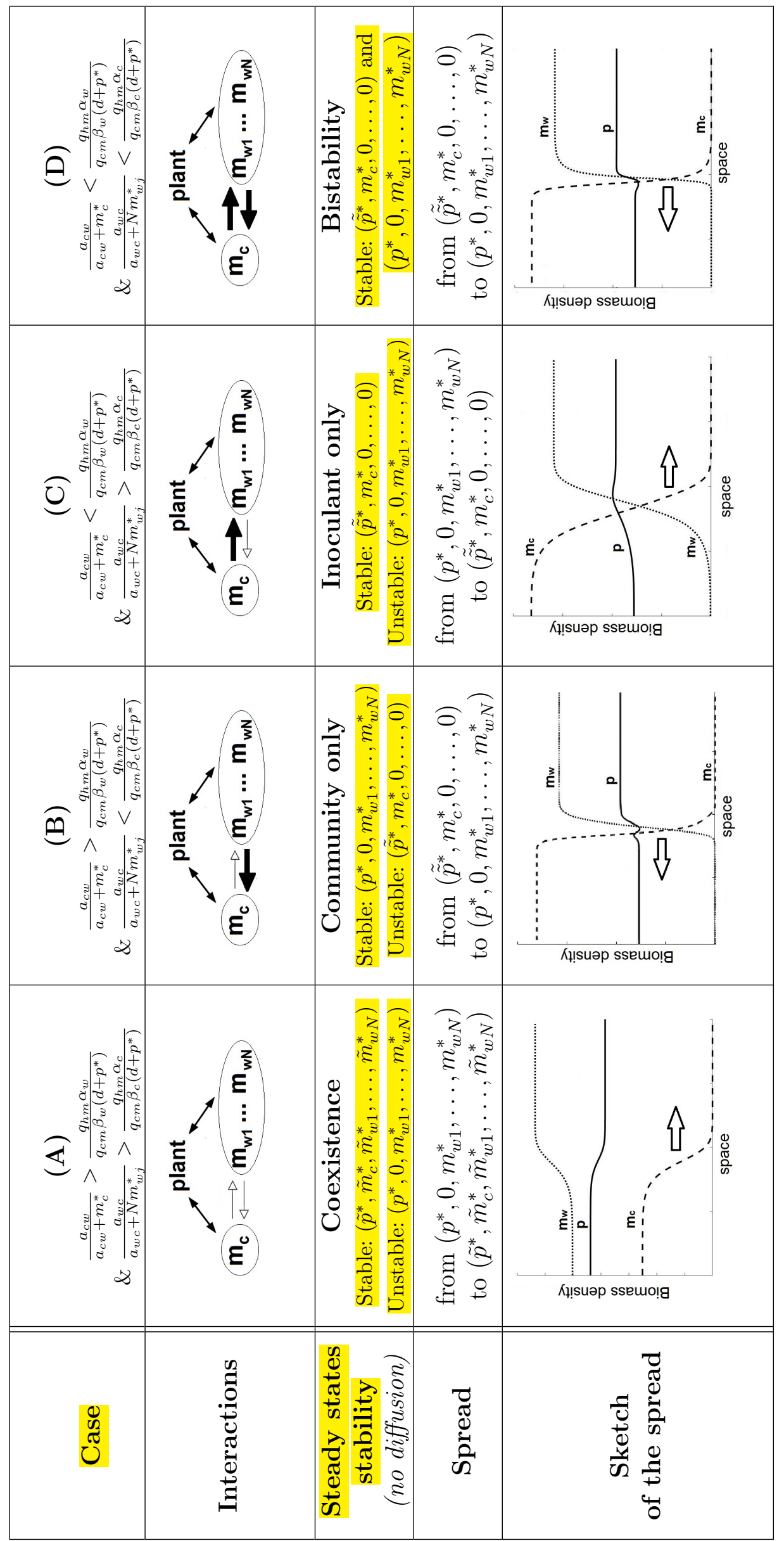


Case (Da) : The inoculant does not persist
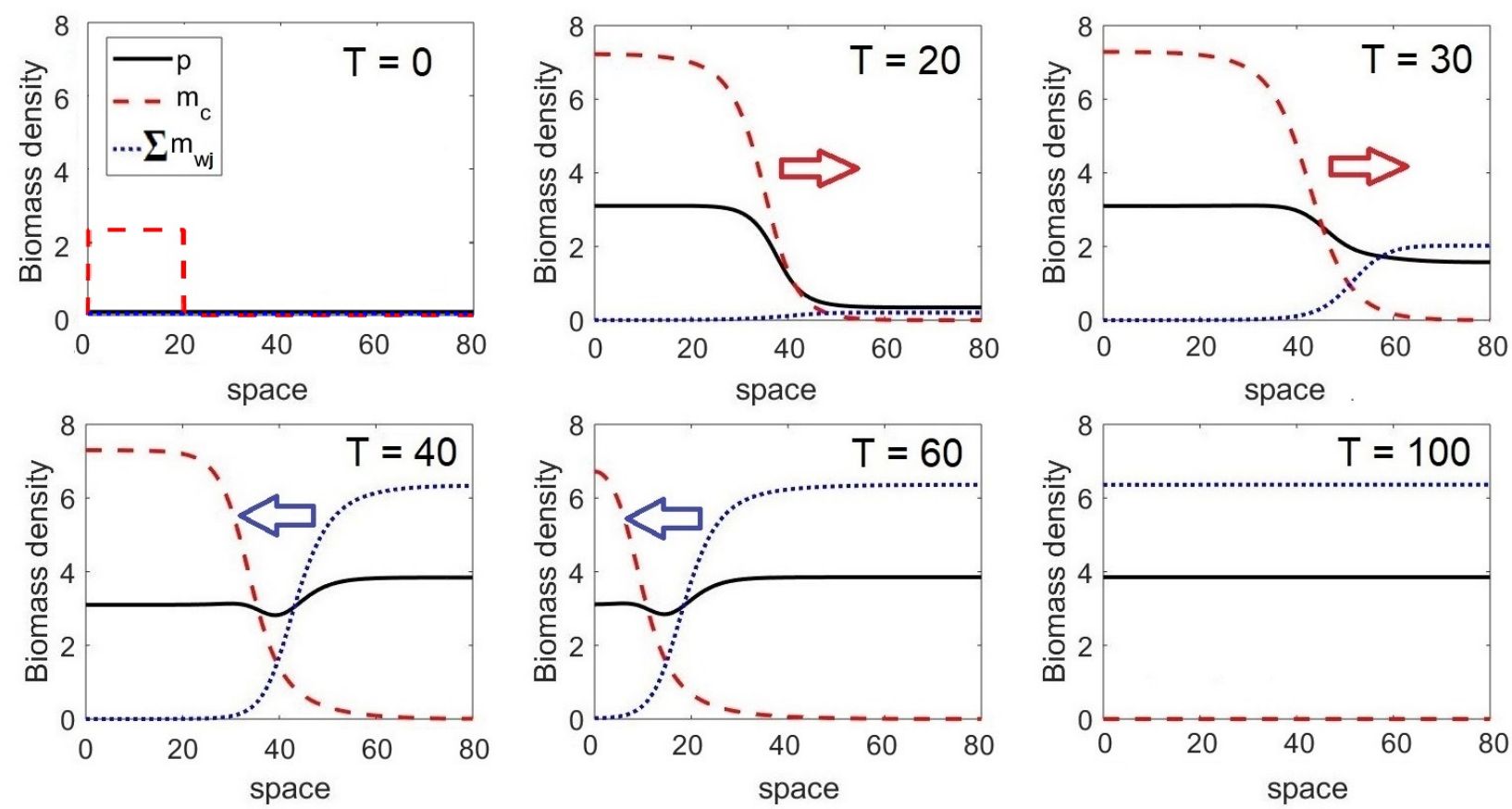

\section{Case (Db): Invasion of the inoculant}
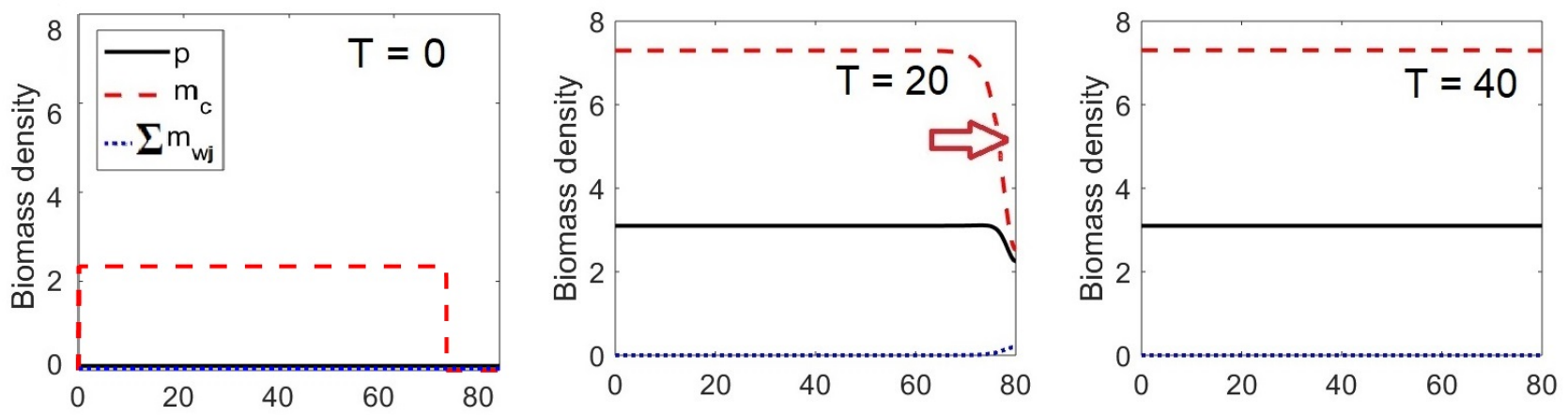

Fig. 1: Spatio-temporal dynamics observed when propagule pressure is high, the density of resident fungi is small, and competition between the inoculant $\left(m_{c}\right)$ and the resident fungal community $\left(\sum m_{w j}\right.$, $j=1,2$ ) is strong (Case (D)). In case (Da), the inoculant initially occupies a small proportion of the whole field, while in $(\mathrm{Db})$ the inoculant initially covers a large part of the field. Competition parameters are $a_{w w}=2.2, a_{w c}=a_{c w}=0.3$. Other model parameters are $q_{h p}=3, q_{c m}=2, q_{h m}=q_{c p}=1$, $\alpha_{w}=\beta_{w}=\alpha_{c}=\beta_{c}=0.4, \mu_{p}=\mu_{m}=0.3, d=1.2, r_{p}=0.02, D_{c}=D_{w_{j}}=0.3, N=2$. 

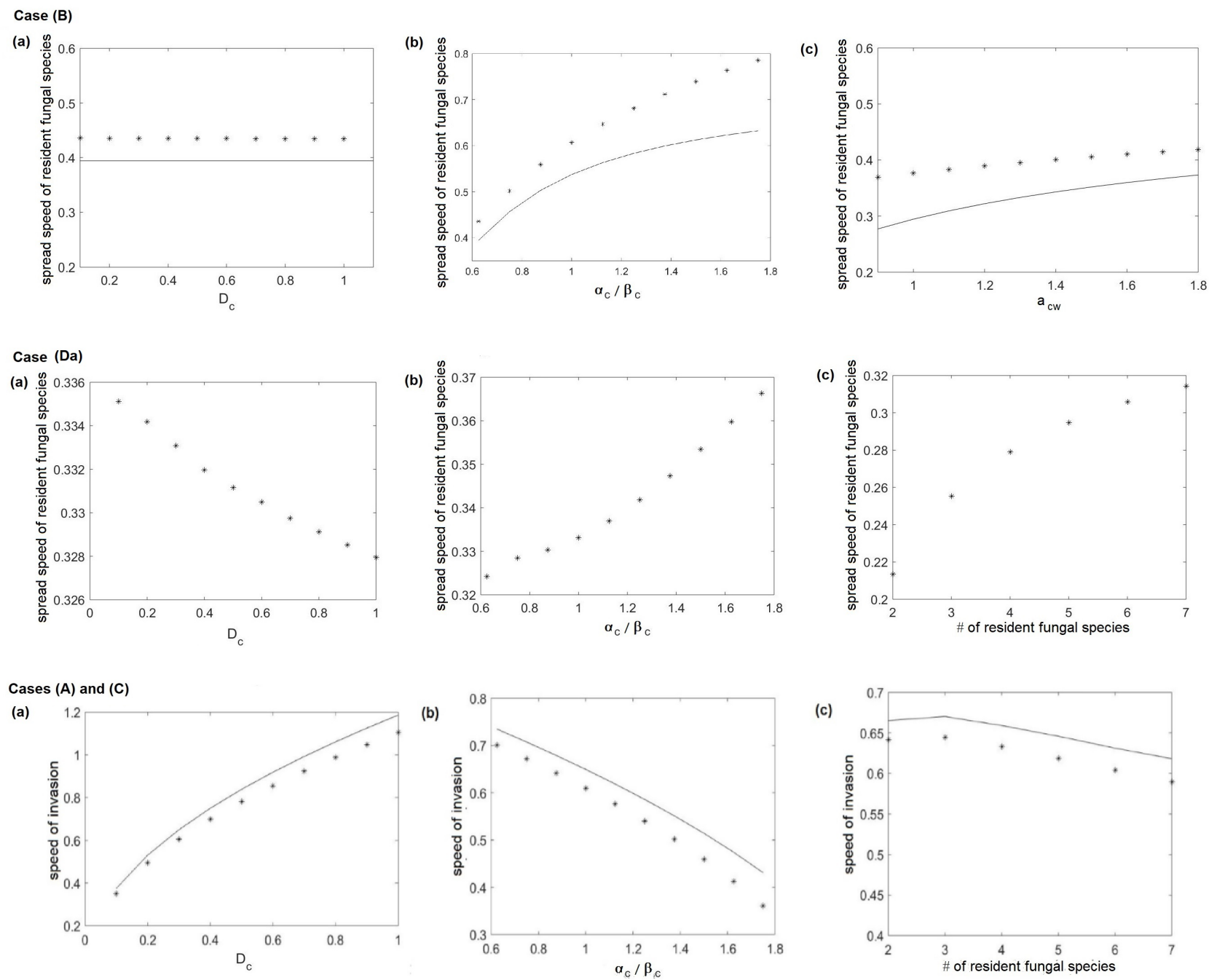

Fig. 2: Speed at which the inoculant is displaced by resident fungal species (cases (B) and (Da)) and speed of invasion of the inoculant (cases (A) and (C)) as a function of the diffusive ability and mutualist quality of the inoculant, of the competitive ability of the inoculant towards resident fungal species, and of the number of species present in the resident fungal community. Solid lines are the theoretically predicted lower bounds (see Eq. (5) for case (B), and Eq. (4) for cases (A) and (C)). The scattered plots are the results of the numerical simulations. Note that the speed obtained in cases (A) and $(\mathrm{C})$ is identical, i.e., the speed of invasion does not depend on whether the inoculant coexist with the resident community (case (A)) or displace the resident community (case (C)). Standard parameter values correspond to those of Fig. 1. Competition parameters are $a_{w w}=a_{w c}=a_{c w}=2.2$, for case (A). $a_{w w}=a_{c w}=2.2, a_{w c}=0.3$ for case (B). $a_{w w}=a_{w c} 2.2$ and $a_{c w}=0.3$ for case (C), plots (a) and (b). $a_{w w}=a_{w c}=3.0, a_{c w}=0.1, \alpha_{w}=0.7$ and $\beta_{w}=0.3$ for case (C), plot (c). $a_{w w}=2.2$, $a_{w c}=a_{c w}=0.3$ for case (Da), plots (a) and (b). $a_{w w}=3, a_{w c}=a_{c w}=0.1$, for case (Da), plot (c). 
(a)
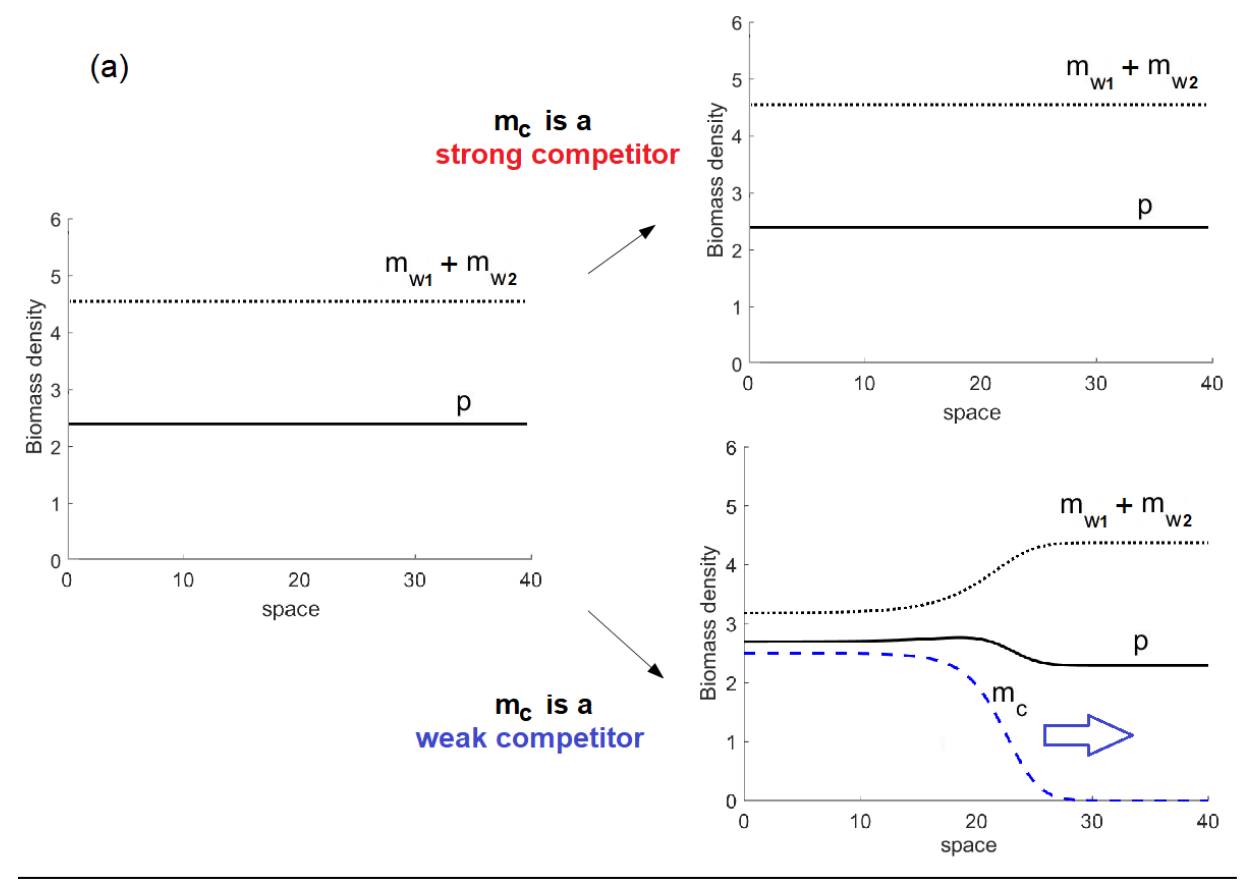

$\mathrm{m}_{\mathrm{c}}$ is a strong competitor

(b)
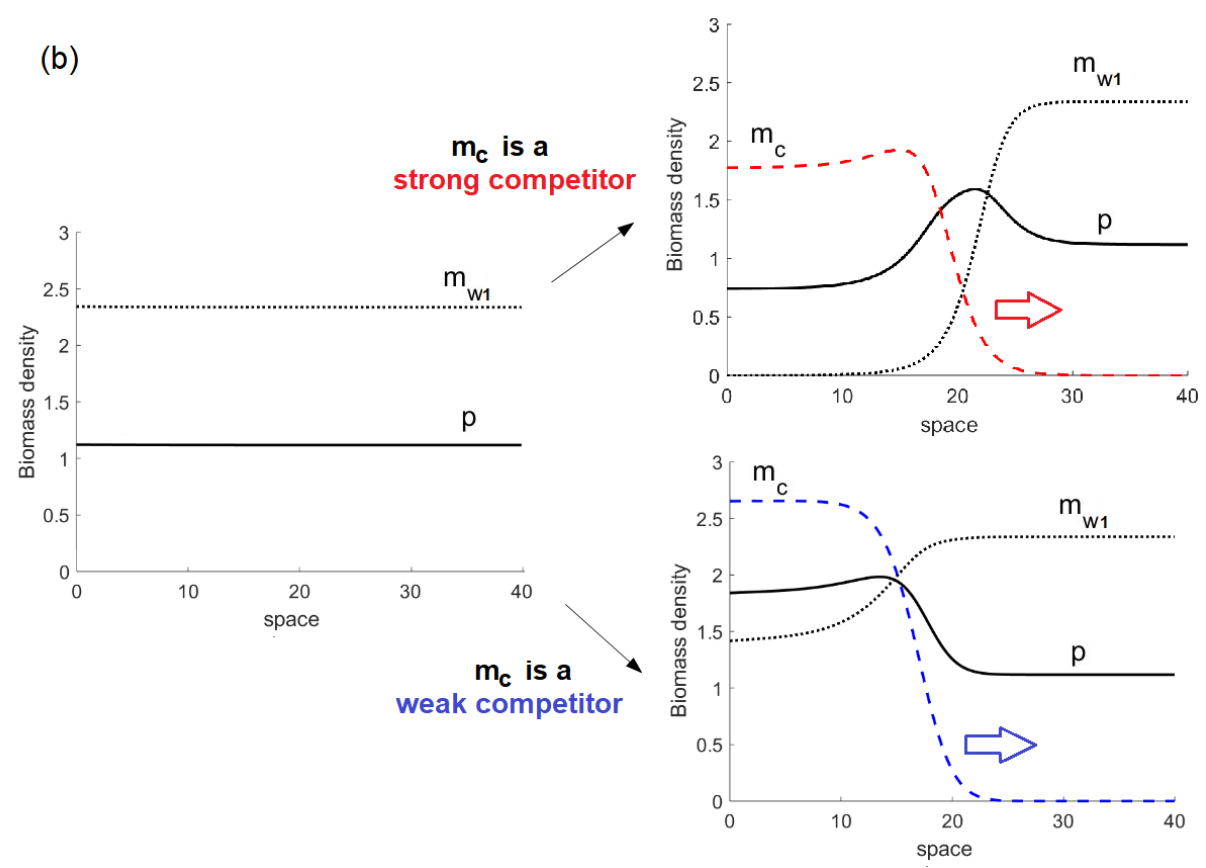

Fig. 3: Simulation showing the dispersal of the inoculant in a field consisting of (a) two or (b) one resident fungal species. We assume that a field inhabited by one or more resident fungi (see figures on the left) is next to a field containing only the inoculant. The figures on the right show how the inoculant will disperse in the field if the inoculant is a strong competitor and a bad mutualist ((a) and (b), top right figures) or a weak competitor ((a) and (b), bottom right figures). In case (a), the resident fungal community is at high density and consists of two fungi. (Note that we show the two-fungi case for simplicity; the result would be qualitatively the same if there were more than two species, i.e., $N>2$ ). In case (b), the resident fungal community is at low density and consists of just one fungus. The biomass density of the strongly competing inoculant is indicated by the dashed red line, while the biomass density of the weakly competing inoculant is shown by the dashed blue line. Competition parameters are $a_{w c}=a_{c w}=0.3$ for the strongly competing inoculant, and $a_{w c}=a_{c w}=2.2$ for the weakly competing inoculant. The mutualist quality of the inoculant is assumed to be lower than the mutualist quality of resident fungal species, where $\alpha_{c}=0.35$ and $\beta_{c}=0.45$, while $\alpha_{w}=0.4$ and $\beta_{w}=0.4$. Other parameters correspond to those of Fig. 1. 

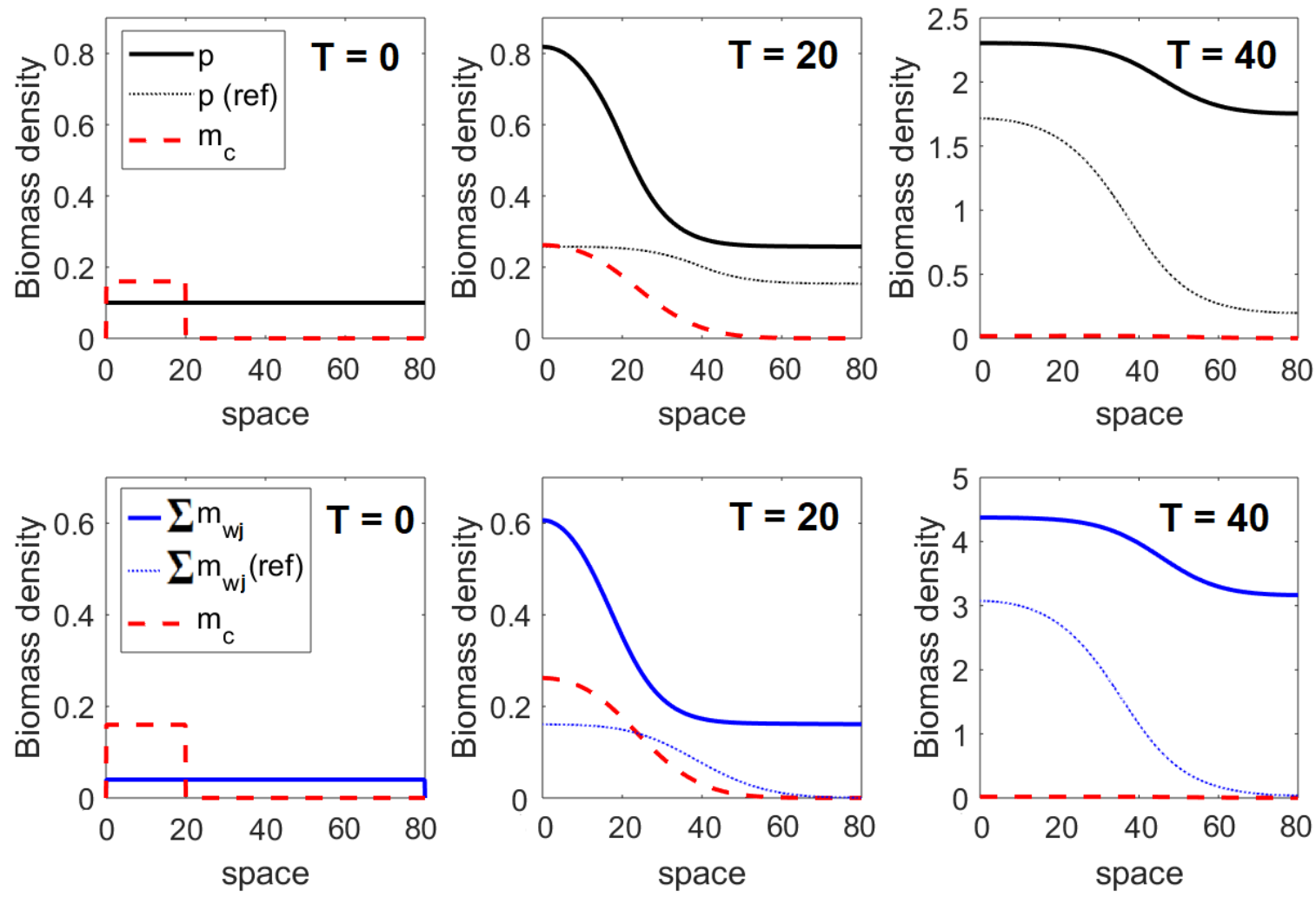

Fig. 4: Spatio-temporal dynamics observed when the inoculant $\left(m_{c}\right)$ is a weak competitor and is eventually displaced by the resident community $\left(\sum m_{w j}, j=1,2\right)$ (Case $\left.(\mathrm{B})\right)$. The dashed red line represents the biomass density of the inoculant. The top row represents plant growth in the presence (solid black line) or absence (dotted black line) of the inoculant. The bottom row represents resident fungal density in the presence (solid blue line) or absence (dotted blue line) of the inoculant. Model parameters used for the simulation correspond to those for Fig. 1, except for the competition parameters that are $a_{w w}=a_{c w}=2.2, a_{w c}=0.3$.

\section{References}

Acosta, A. L., Giannini, T. C., Imperatriz-Fonseca, V. L., and Saraiva, A. M. (2016). Worldwide alien invasion: A methodological approach to forecast the potential spread of a highly invasive pollinator. PLoS one, 11(2):e0148295.

Baum, C., El-Tohamy, W., and Gruda, N. (2015). Increasing the productivity and product quality of vegetable crops using arbuscular mycorrhizal fungi: A review. Scientia Horticulturae, 187:131-141.

Bender, S. F., Schlaeppi, K., Held, A., and Van der Heijden, M. G. (2019). Establishment success and crop growth effects of an arbuscular mycorrhizal fungus inoculated into swiss corn fields. Agriculture, Ecosystems \& Environment, 273:13-24.

Berruti, A., Lumini, E., Balestrini, R., and Bianciotto, V. (2016). Arbuscular mycorrhizal fungi as natural biofertilizers: let's benefit from past successes. Frontiers in Microbiology, $6: 1559$.

Bever, J. D. (2002). Host-specificity of am fungal population growth rates can generate feedback on plant growth. Plant and Soil, 244(1-2):281-290. 
Burie, J.-B., Calonnec, A., and Langlais, M. (2008). Modeling of the invasion of a fungal disease over a vineyard. In Mathematical Modeling of Biological Systems, Volume II, pages 11-21. Springer.

Buysens, C., César, V., Ferrais, F., de Boulois, H. D., and Declerck, S. (2016). Inoculation of medicago sativa cover crop with rhizophagus irregularis and trichoderma harzianum increases the yield of subsequently-grown potato under low nutrient conditions. Applied Soil Ecology, 105:137-143.

Calvet, C., Camprubi, A., Pérez-Hernández, A., and Lovato, P. E. (2013). Plant growth stimulation and root colonization potential of in vivo versus in vitro arbuscular mycorrhizal inocula. HortScience, 48(7):897-901.

Ceballos, I., Ruiz, M., Fernández, C., Peña, R., Rodríguez, A., and Sanders, I. R. (2013). The in vitro mass-produced model mycorrhizal fungus, rhizophagus irregularis, significantly increases yields of the globally important food security crop cassava. PLoS One, 8(8):e70633.

Curaqueo, G., Barea, J. M., Acevedo, E., Rubio, R., Cornejo, P., and Borie, F. (2011). Effects of different tillage system on arbuscular mycorrhizal fungal propagules and physical properties in a mediterranean agroecosystem in central chile. Soil and Tillage Research, 113(1):11-18.

Davison, J., Öpik, M., Zobel, M., Vasar, M., Metsis, M., and Moora, M. (2012). Communities of arbuscular mycorrhizal fungi detected in forest soil are spatially heterogeneous but do not vary throughout the growing season. PloS One, 7(8).

del Mar Alguacil, M., Torrecillas, E., Kohler, J., and Roldán, A. (2011). A molecular approach to ascertain the success of "in situ" am fungi inoculation in the revegetation of a semiarid, degraded land. Science of the Total Environment, 409(15):2874-2880.

Dickie, I. A., Nuñez, M. A., Pringle, A., Lebel, T., Tourtellot, S. G., and Johnston, P. R. (2016). Towards management of invasive ectomycorrhizal fungi. Biological Invasions, 18(12):3383-3395.

Emam, T. (2016). Local soil, but not commercial amf inoculum, increases native and nonnative grass growth at a mine restoration site. Restoration Ecology, 24(1):35-44.

Farmer, M., Li, X., Feng, G., Zhao, B., Chatagnier, O., Gianinazzi, S., Gianinazzi-Pearson, V., and Van Tuinen, D. (2007). Molecular monitoring of field-inoculated amf to evaluate persistence in sweet potato crops in china. Applied Soil Ecology, 35(3):599-609.

Friese, C. F. and Allen, M. F. (1991). The spread of va mycorrhizal fungal hyphae in the soil: inoculum types and external hyphal architecture. Mycologia, 83(4):409-418.

Gianinazzi, S. and Vosátka, M. (2004). Inoculum of arbuscular mycorrhizal fungi for production systems: science meets business. Canadian Journal of Botany, 82(8):1264-1271.

Girardin, L. (2017). Non-cooperative fisher-kpp systems: traveling waves and long-time behavior. Nonlinearity, 31(1):108.

Goldstein, J., Park, J., Haran, M., Liebhold, A., and Bjørnstad, O. N. (2019). Quantifying spatio-temporal variation of invasion spread. Proceedings of the Royal Society B, 286(1894):20182294. 
Gosling, P., Jones, J., and Bending, G. D. (2016). Evidence for functional redundancy in arbuscular mycorrhizal fungi and implications for agroecosystem management. Mycorrhiza, 26(1):77-83.

Guo, J.-S., Nakamura, K.-I., Ogiwara, T., and Wu, C.-H. (2019). The sign of traveling wave speed in bistable dynamics. Discrete $\&$ Continuous Dynamical Systems-A.

Haller, B. C. (2014). Theoretical and empirical perspectives in ecology and evolution: a survey. Bioscience, 64(10):907-916.

Hamel, C. and Smith, D. L. (1991). Plant development in a mycorrhizal field-grown mixture. Soil Biology and Biochemistry, 23(7):661-665.

Hart, M. M., Antunes, P. M., and Abbott, L. K. (2017). Unknown risks to soil biodiversity from commercial fungal inoculants. Nature Ecology \& Evolution, 1(4):0115.

Hart, M. M., Antunes, P. M., Chaudhary, V. B., and Abbott, L. K. (2018). Fungal inoculants in the field: Is the reward greater than the risk? Functional Ecology, 32(1):126-135.

Herbold, B. and Moyle, P. B. (1986). Introduced species and vacant niches. The American Naturalist, 128(5):751-760.

Hijri, M. (2016). Analysis of a large dataset of mycorrhiza inoculation field trials on potato shows highly significant increases in yield. Mycorrhiza, 26(3):209-214.

Holland, J. N. and DeAngelis, D. L. (2010). A consumer-resource approach to the densitydependent population dynamics of mutualism. Ecology, 91(5):1286-1295.

Holzer, M. and Scheel, A. (2012). A slow pushed front in a lotka-volterra competition model. Nonlinearity, 25(7):2151.

Hosono, Y. (1998). The minimal speed of traveling fronts for a diffusive lotka-volterra competition model. Bulletin of Mathematical Biology, 60(3):435-448.

Huang, W. (2010). Problem on minimum wave speed for a lotka-volterra reaction-diffusion competition model. Journal of Dynamics and Differential Equations, 22(2):285-297.

Huang, W. and Han, M. (2011). Non-linear determinacy of minimum wave speed for a lotkavolterra competition model. Journal of Differential Equations, 251(6):1549-1561.

Jeffries, P., Gianinazzi, S., Perotto, S., Turnau, K., and Barea, J.-M. (2003). The contribution of arbuscular mycorrhizal fungi in sustainable maintenance of plant health and soil fertility. Biology and Fertility of Soils, 37(1):1-16.

Jin, H., G., J. J., and Walley, F. L. (2013). Impact of arbuscular mycorrhizal fungal inoculants on subsequent arbuscular mycorrhizal fungi colonization in pot-cultured field pea (pisum sativum 1.). Mycorrhiza, 23(1):45-59.

Jørgensen, S. (1994). Models as instruments for combination of ecological theory and environmental practice. Ecological Modelling, 75:5-20.

Karasawa, T. and Takebe, M. (2012). Temporal or spatial arrangements of cover crops to promote arbuscular mycorrhizal colonization and p uptake of upland crops grown after nonmycorrhizal crops. Plant and Soil, 353(1-2):355-366. 
Kinezaki, N., Kawasaki, K., Takasu, F., and Shigesada, N. (2003). Modeling biological invasions into periodically fragmented environments. Theoretical Population Biology, 64(3):291302.

Koch, A. M., Antunes, P. M., Barto, E. K., Cipollini, D., Mummey, D. L., and Klironomos, J. N. (2011). The effects of arbuscular mycorrhizal (am) fungal and garlic mustard introductions on native am fungal diversity. Biological Invasions, 13(7):1627-1639.

Köhl, L., Lukasiewicz, C. E., and Van der Heijden, M. G. (2016). Establishment and effectiveness of inoculated arbuscular mycorrhizal fungi in agricultural soils. Plant, Cell $\mathscr{E}$ Environment, 39(1):136-146.

Kokkoris, V., Li, Y., Hamel, C., Hanson, K., and Hart, M. (2019a). Site specificity in establishment of a commercial arbuscular mycorrhizal fungal inoculant. Science of The Total Environment, 660:1135-1143.

Kokkoris, V., Miles, T., and Hart, M. M. (2019b). The role of in vitro cultivation on asymbiotic trait variation in a single species of arbuscular mycorrhizal fungus. Fungal Biology, 123(4):307-317.

Latef, A. A. H. A., Hashem, A., Rasool, S., Abd_Allah, E. F., Alqarawi, A., Egamberdieva, D., Jan, S., Anjum, N. A., and Ahmad, P. (2016). Arbuscular mycorrhizal symbiosis and abiotic stress in plants: A review. Journal of Plant Biology, 59(5):407-426.

Lekberg, Y. and Koide, R. (2005). Is plant performance limited by abundance of arbuscular mycorrhizal fungi? a meta-analysis of studies published between 1988 and 2003. New Phytologist, 168(1):189-204.

Lekevičius, E. (2009). Vacant niches in nature, ecology, and evolutionary theory: a minireview. Ekologija, 55(3-4):165-74.

Lewis, M. A., Li, B., and Weinberger, H. F. (2002). Spreading speed and linear determinacy for two-species competition models. Journal of Mathematical Biology, 45(3):219-233.

Li, B., Weinberger, H. F., and Lewis, M. A. (2005). Spreading speeds as slowest wave speeds for cooperative systems. Mathematical biosciences, 196(1):82-98.

Litchman, E. (2010). Invisible invaders: non-pathogenic invasive microbes in aquatic and terrestrial ecosystems. Ecology Letters, 13(12):1560-1572.

Loján, P., Senés-Guerrero, C., Suárez, J. P., Kromann, P., Schüßler, A., and Declerck, S. (2017). Potato field-inoculation in ecuador with rhizophagus irregularis: no impact on growth performance and associated arbuscular mycorrhizal fungal communities. Symbiosis, $73(1): 45-56$.

MacArthur, R. and Levins, R. (1967). The limiting similarity, convergence, and divergence of coexisting species. The American Naturalist, 101(921):377-385.

Mallon, C. A., Van Elsas, J. D., and Salles, J. F. (2015). Microbial invasions: the process, patterns, and mechanisms. Trends in microbiology, 23(11):719-729.

Martignoni, M. M., Hart, M. M., Garnier, J., and Tyson, R. C. (2020a). Parasitism within mutualist guilds explains the maintenance of diversity in multi-species mutualisms. Theoretical Ecology, pages 1-13. 
Martignoni, M. M., Hart, M. M., Tyson, R. C., and Garnier, J. (2020b). Diversity within mutualist guilds promotes coexistence and reduces the risk of invasion from an alien mutualist. Proceedings of the Royal Society B, 287(1923):20192312.

Mummey, D. L., Antunes, P. M., and Rillig, M. C. (2009). Arbuscular mycorrhizal fungi pre-inoculant identity determines community composition in roots. Soil Biology and Biochemistry, 41(6):1173-1179.

Murray, J. D. (1993). Biological Waves: Multi-Species Reaction Diffusion Models, pages 311359. Springer, Berlin, Heidelberg.

Niwa, R., Koyama, T., Sato, T., Adachi, K., Tawaraya, K., Sato, S., Hirakawa, H., Yoshida, S., and Ezawa, T. (2018). Dissection of niche competition between introduced and indigenous arbuscular mycorrhizal fungi with respect to soybean yield responses. Scientific Reports, 8.

Odenbaugh, J. (2005). Idealized, inaccurate but successful: A pragmatic approach to evaluating models in theoretical ecology. Biology and Philosophy, 20(2-3):231-255.

Okubo, A., Maini, P. K., Williamson, M. H., and Murray, J. D. (1989). On the spatial spread of the grey squirrel in britain. Proceedings of the Royal Society of London. B. Biological Sciences, 238(1291):113-125.

Omirou, M., Fasoula, D. A., and Ioannides, I. M. (2016). Bradyrhizobium inoculation alters indigenous amf community assemblages and interacts positively with amf inoculum to improve cowpea performance. Applied Soil Ecology, 108:381-389.

Peay, K. G. and Bruns, T. D. (2014). Spore dispersal of basidiomycete fungi at the landscape scale is driven by stochastic and deterministic processes and generates variability in plantfungal interactions. New Phytologist, 204(1):180-191.

Peay, K. G., Schubert, M. G., Nguyen, N. H., and Bruns, T. D. (2012). Measuring ectomycorrhizal fungal dispersal: macroecological patterns driven by microscopic propagules. Molecular Ecology, 21(16):4122-4136.

Pellegrino, E., Bedini, S., Avio, L., Bonari, E., and Giovannetti, M. (2011). Field inoculation effectiveness of native and exotic arbuscular mycorrhizal fungi in a mediterranean agricultural soil. Soil Biology and Biochemistry, 43(2):367-376.

Pellegrino, E., Turrini, A., Gamper, H. A., Cafà, G., Bonari, E., Young, J. P. W., and Giovannetti, M. (2012). Establishment, persistence and effectiveness of arbuscular mycorrhizal fungal inoculants in the field revealed using molecular genetic tracing and measurement of yield components. New Phytologist, 194(3):810-822.

Pozo, M. J. and Azcón-Aguilar, C. (2007). Unraveling mycorrhiza-induced resistance. Current Opinion in Plant Biology, 10(4):393-398.

Real, L. A. and Biek, R. (2007). Spatial dynamics and genetics of infectious diseases on heterogeneous landscapes. Journal of the Royal Society Interface, 4(16):935-948.

Ricciardi, A., Blackburn, T. M., Carlton, J. T., Dick, J. T., Hulme, P. E., Iacarella, J. C., Jeschke, J. M., Liebhold, A. M., Lockwood, J. L., MacIsaac, H. J., et al. (2017). Invasion science: a horizon scan of emerging challenges and opportunities. Trends in Ecology 85 Evolution, 32(6):464-474. 
Roques, L., Hosono, Y., Bonnefon, O., and Boivin, T. (2015). The effect of competition on the neutral intraspecific diversity of invasive species. Journal of Mathematical Biology, 71(2):465-489.

Ryan, M. H. and Graham, J. H. (2002). Is there a role for arbuscular mycorrhizal fungi in production agriculture? Plant and Soil, 244(1-2):263-271.

Säle, V., Aguilera, P., Laczko, E., Mäder, P., Berner, A., Zihlmann, U., van der Heijden, M. G., and Oehl, F. (2015). Impact of conservation tillage and organic farming on the diversity of arbuscular mycorrhizal fungi. Soil Biology and Biochemistry, 84:38-52.

Schnoor, T. K., Lekberg, Y., Rosendahl, S., and Olsson, P. A. (2011). Mechanical soil disturbance as a determinant of arbuscular mycorrhizal fungal communities in semi-natural grassland. Mycorrhiza, 21(3):211-220.

Schwartz, M. W., Hoeksema, J. D., Gehring, C. A., Johnson, N. C., Klironomos, J. N., Abbott, L. K., and Pringle, A. (2006). The promise and the potential consequences of the global transport of mycorrhizal fungal inoculum. Ecology Letters, 9(5):501-515.

Smith, S. E. and Read, D. J. (2010). Mycorrhizal symbiosis. Academic Press.

Soubeyrand, S., Held, L., Höhle, M., and Sache, I. (2008). Modelling the spread in space and time of an airborne plant disease. Journal of the Royal Statistical Society: Series C (Applied Statistics), 57(3):253-272.

Streeter, J. G. (1994). Failure of inoculant rhizobia to overcome the dominance of indigenous strains for nodule formation. Canadian Journal of Microbiology, 40(7):513-522.

Sỳkorová, Z., Börstler, B., Zvolenská, S., Fehrer, J., Gryndler, M., Vosátka, M., and Redecker, D. (2012). Long-term tracing of rhizophagus irregularis isolate beg140 inoculated on phalaris arundinacea in a coal mine spoil bank, using mitochondrial large subunit rdna markers. Mycorrhiza, 22(1):69-80.

Symanczik, S., Courty, P.-E., Boller, T., Wiemken, A., and Al-Yahya'ei, M. N. (2015). Impact of water regimes on an experimental community of four desert arbuscular mycorrhizal fungal (amf) species, as affected by the introduction of a non-native amf species. Mycorrhiza, 25(8):639-647.

Thiet, R. K. and Boerner, R. (2007). Spatial patterns of ectomycorrhizal fungal inoculum in arbuscular mycorrhizal barrens communities: implications for controlling invasion by pinus virginiana. Mycorrhiza, 17(6):507-517.

Thomsen, C. N. and Hart, M. M. (2018). Using invasion theory to predict the fate of arbuscular mycorrhizal fungal inoculants. Biological Invasions, 20(10):2695-2706.

Tyson, R., Stern, L., and LeVeque, R. J. (2000). Fractional step methods applied to a chemotaxis model. Journal of Mathematical Biology, 41(5):455-475.

Vellinga, E. C., Wolfe, B. E., and Pringle, A. (2009). Global patterns of ectomycorrhizal introductions. New Phytologist, 181(4):960-973.

Verbruggen, E., van der Heijden, M. G., Rillig, M. C., and Kiers, E. T. (2013). Mycorrhizal fungal establishment in agricultural soils: factors determining inoculation success. New Phytologist, 197(4):1104-1109. 
628

Wagg, C., Barendregt, C., Jansa, J., and Heijden, M. G. (2015). Complementarity in both plant and mycorrhizal fungal communities are not necessarily increased by diversity in the other. Journal of Ecology, 103(5):1233-1244.

Warner, N. J., Allen, M. F., and MacMahon, J. A. (1987). Dispersal agents of vesiculararbuscular mycorrhizal fungi in a disturbed arid ecosystem. Mycologia, 79(5):721-730.

Zhang, S., Lehmann, A., Zheng, W., You, Z., and Rillig, M. C. (2019). Arbuscular mycorrhizal fungi increase grain yields: a meta-analysis. New Phytologist, 222(1):543-555. 


\section{Supplementary information}

\section{A model for investigating the persistence and spread of fungal resident}

To investigate the persistence and spread of the introduction of a commercial fungal inoculant $\left(m_{c}\right)$ into a field inhabited by a resident fungal community $\left(m_{w_{j}}\right.$, where $\left.j=1 . . N\right)$, we extend the model developed by Martignoni et al. (2020b) to explicitly consider random fungal dispersal. In the model, plant and fungal coupled growth is determined by the density dependent functions $f_{p}\left(p, m_{c}, m_{w_{j}}\right)$, for plant growth, $f_{m_{c}}\left(p, m_{c}, m_{w_{j}}\right)$, for inoculum growth, and $f_{m_{w_{j}}}\left(p, m_{c}, m_{w_{j}}\right)$, for the growth of the resident fungal community, defined in Martignoni et al. (2020b). The complete model can be written as

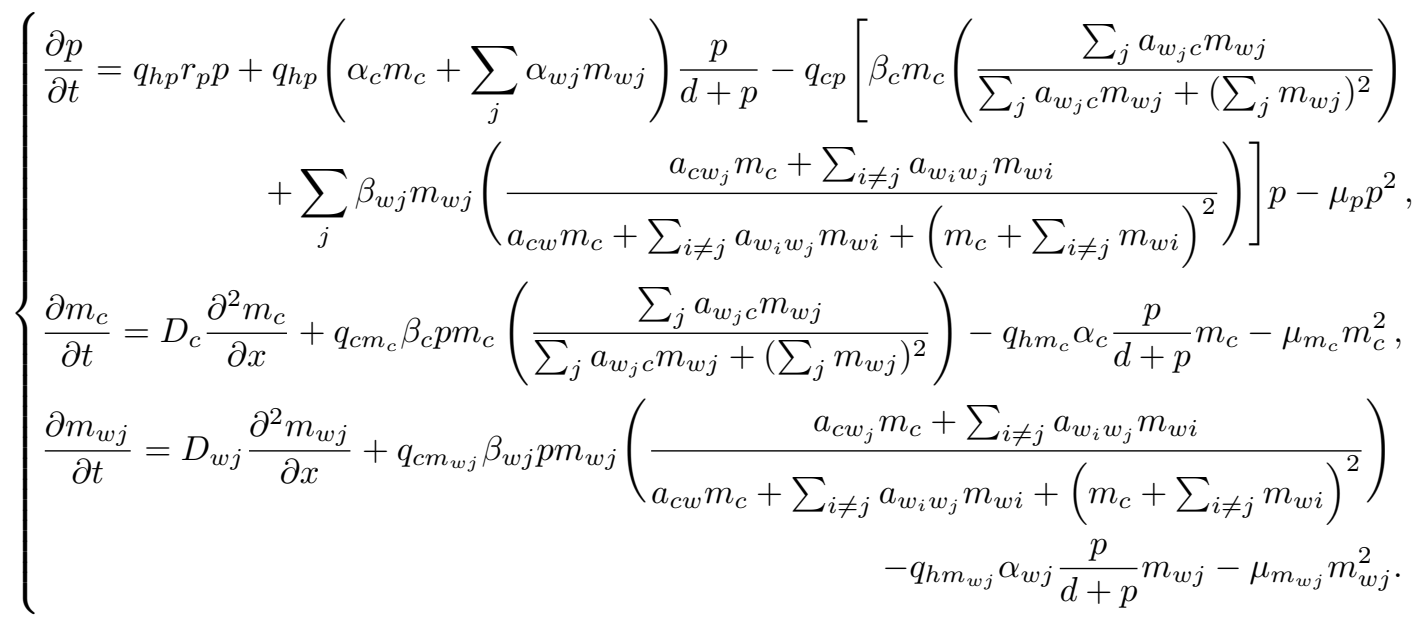

A description of the model parameters with respective measurement unit is provided in Table S1.

To simplify the analysis, we will assume that the competition strength between the commercial fungus $m_{c}$ and individuals of the wild community $m_{w j}$ and between individuals of the wild community are the same for all fungi (i.e. $a_{w_{j} c}=a_{w c}, a_{c w_{j}}=a_{c w}$ and $a_{w_{i} w_{j}}=a_{w w}$ ). We will further assume that all fungal parameters are the same, except for the phosphorus and carbon exchange ability ( $\alpha$ 's and $\beta$ 's parameters). With these simplifications, the model of Eq. (6) becomes

$$
\left\{\begin{array}{c}
\frac{\partial p}{\partial t}=q_{h p} r_{p} p+q_{h p}\left(\alpha_{c} m_{c}+N \alpha_{w} m_{w j}\right) \frac{p}{d+p}-q_{c p}\left[\beta_{c} m_{c} \frac{a_{w c}}{a_{w c}+N m_{w j}}+\right. \\
\left.N\left(\beta_{w} m_{w j} \frac{a_{c w} m_{c}+a_{w w}(N-1) m_{w j}}{a_{c w} m_{c}+a_{w w}(N-1) m_{w j}+\left(m_{c}+(N-1) m_{w j}\right)^{2}}\right)\right] p-\mu_{p} p^{2} \\
\frac{\partial m_{c}}{\partial t}=D_{c} \frac{\partial^{2} m_{c}}{\partial x^{2}}+q_{c m} \beta_{c} p m_{c}\left(\frac{a_{w c}}{a_{w c}+N m_{w j}}\right)-q_{h m} \alpha_{c} \frac{p}{d+p} m_{c}-\mu_{m} m_{c}^{2}, \\
\frac{\partial m_{w j}}{\partial t}=D_{w} \frac{\partial^{2} m_{w j}}{\partial x^{2}}+q_{c m} \beta_{w} p m_{w j}\left(\frac{a_{c w} m_{c}+a_{w w}(N-1) m_{w j}}{a_{c w} m_{c}+a_{w w}(N-1) m_{w j}+\left(m_{c}+(N-1) m_{w j}\right)^{2}}\right) \\
-q_{h m} \alpha_{w} \frac{p}{d+p} m_{w j}-\mu_{m} m_{w j}^{2}
\end{array} .\right.
$$


We will use Eq. (7) to study how the competition strength between the inoculant and resident fungal species affect fungal spread. We will consider a community of coexisting weakly competing fungi (i.e., $a_{w w}$ is large), competing with the inoculated fungal species $m_{c}$. The scenarios considered are sketched in Table. 2 . In case (A), competition between the inoculant and the resident community is weak (i.e., $a_{w c}$ and $a_{c w}$ are large). In cases (B) and $(\mathrm{C})$ competition between the inoculant and the resident community is asymmetric, where either the inoculant or the resident community is a superior competitor (i.e., $a_{c w}>a_{w c}$ or $a_{c w}<a_{w c}$ ). In case (D) competition between the inoculant and the resident fungal community is strong (i.e., $a_{w c}$ and $a_{c w}$ are small). Additionally, we will determine how the spreading speed is affected by the dispersal ability of the inoculant (parameter $D_{c}$ ), by its mutualist quality $\left(\alpha_{c} / \beta_{c}\right.$ ratio), and by the number of fungal species present in the resident community.

Model outputs will be analysed through traveling wave analysis and numerical simulations. As discussed in the manuscript, the output of case (D) depends on the initial distribution of inoculant; in case (Da) the inoculant is introduced over a small proportion of the field, while in case $(\mathrm{Db})$ the inoculant is introduced over a large proportion of the field. In our traveling wave analysis, we will be looking at the spread of inoculants from a region with positive inoculant density into a region where the inoculant is absent (see boundary conditions given in Eq. (10)). These initial distribution of inoculants allows us to investigate case (Da) analytically. Case (Db) is analytically intractable. Numerical simulations are run in Matlab R2017a. Solutions are found by using a semi-implicit scheme (Tyson et al., 2000), where the reaction term is solved explicitly and the diffusion term is solved implicitly. The discretization used for the space is $\mathrm{d} x=0.05$, and the discretion used for the time is $\mathrm{d} t=0.05$.

\section{Model analysis}

We are interested in understanding whether the introduction of a new fungal species $\left(m_{c}\right)$ in the presence of an existing fungal community $\left(m_{w j}\right)$ will result in a spread of the new species into the field. To tackle this problem, we will look at travelling wave solution of Eq. (7), which are particular solutions describing the invasion of one steady state by an other one at constant speed $c$. More precisely, we assume that the solution of Eq. (7) are of the form $p(t, x)=P(x-c t)=P(z), m_{c}(t, x)=M_{c}(x-c t)=M_{c}(z)$ and $m_{w j}(t, x)=M_{w j}(x-c t)=$ $M_{w j}(z)$ for an unknown speed $c \in \mathbb{R}$. By replacing these expressions into Eq. (7) we obtain

$$
\left\{\begin{aligned}
-c \frac{d P}{d z} & =F_{P}\left(P, M_{c}, M_{w j}\right), \\
-c \frac{d M_{c}}{d z} & =D_{c} \frac{d^{2} M_{c}}{d z^{2}}+F_{M_{c}}\left(P, M_{c}, M_{w j}\right), \\
-c \frac{d M_{w j}}{d z} & =D_{w} \frac{d^{2} M_{w j}}{d z^{2}}+F_{M_{w j}}\left(P, M_{c}, M_{w j}\right) .
\end{aligned}\right.
$$

In order to lighten the notations, we define the vector $\mathbf{U}(x-c t)=\mathbf{U}(z)=\left(P, M_{c}, M_{w j}\right)$, the diffusion matrix $\mathbf{D}=\operatorname{diag}\left(0, D_{c}, D_{w j}\right)$ and the interaction function $\mathbf{F}(\mathbf{U}(z))=\left(F_{P}, F_{M_{c}}, F_{M_{w j}}\right)$. Then Eq. (8) can be written as

$$
\mathbf{D U}^{\prime \prime}(z)+c \mathbf{U}^{\prime}(z)+\mathbf{F}(\mathbf{U}(z))=\mathbf{0} .
$$

We will focus on three particular steady states which stability have been already investigated in Martignoni et al. (2020b):

- Community only: $\mathbf{U}_{w}^{*}=\left(P_{w}^{*}, 0, M_{w j}^{*}\right)$, 
- Coexistence: $\mathbf{U}_{c w}^{* *}=\left(P_{c w}^{* *}, M_{c}^{* *}, M_{w j}^{* *}\right)$,

- Inoculant only: $\mathbf{U}_{c}^{*}=\left(P_{c}^{*}, M_{c}^{*}, 0\right)$.

The stability of these steady states depends on the strength of the competitive interactions between the fungi. As shown in Table S2, in case (A), competition between the inoculant and the resident community is weak. In cases (B) and (C) competition between the inoculant and the resident community is asymmetric, where either the inoculant or the resident community is a superior competitor. In case (D) competition between the inoculant and the resident fungal community is strong. From the analysis of the non-spatial model (Martignoni et al., 2020b) we know that

- Case (A): $\mathbf{U}_{c w}^{* *}$ stable, $\mathbf{U}_{w}^{*}$ unstable,

- Case (B): $\mathbf{U}_{c}^{*}$ unstable, $\mathbf{U}_{w}^{*}$ stable,

- Case $(\mathrm{C})$ : $\mathbf{U}_{c}^{*}$ stable, $\mathbf{U}_{w}^{*}$ unstable,

- Case (D): $\mathbf{U}_{c}^{*}$ stable, $\mathbf{U}_{w}^{*}$ stable *

We will consider on the right of the domain a steady state where the inoculant biomass density is zero (i.e. $M_{c}(\infty)=0$ ) and only the resident community and the plant are present. On the left we will assume that the steady state has a positive density of a commercial inoculant $\left(M_{c}(-\infty)>0\right)$, plant biomass density is positive and the biomass density of the resident community can be zero or positive depending on which scenario has been investigated. This situation corresponds to investigating whether the steady state $\mathbf{U}_{w}^{*}$ can be invaded by a steady state with a positive density of the inoculant (i.e. either $\mathbf{U}_{c w}^{* *}$ or $\mathbf{U}_{c}^{*}$ ). Thus the boundary conditions can be expressed as

$$
\mathbf{U}(-\infty)=\left\{\begin{array}{ll}
\mathbf{U}_{c w}^{* *} & \text { Case }(\mathrm{A}) \\
\mathbf{U}_{c}^{*} & \text { Cases }(\mathrm{B})-(\mathrm{Da})
\end{array} \quad \text { and } \quad \mathbf{U}(+\infty)=\mathbf{U}_{w}^{*}\right.
$$

The solutions to Eq. (9) satify boundary conditions for $P, M_{w j}$ and $M_{c}$. The stability of the steady states involved in the boundary conditions, may help us to describe the speed at which the solution moves.

When one stable steady state and one unstable steady state are present (cases (A)-(C)) the stable state will invade the unstable state at constant speed $c$. In this case, there is a monostable traveling waves and one may expect an estimate of the minimal speed of propagation using the linearized problem around the unstable steady state. In case (Da), a bistable traveling wave connects two stable steady state. In this case it is difficult to determine the actual speed of invasion, however, there are some techniques to estimate the sign of the propagation speed, to determine which of steady states will invade the other. We will first analyse the three monostable cases, and then investigate the bistable one. In order to gain more insight into the results of the analysis, We will explore numerically how the invasion speed varies as a function of diversity of the resident community, as well as a function of different characteristic of the inoculant, such as dispersal ability $\left(D_{c}\right)$ and mutualist quality $\left(\alpha_{c} / \beta_{c}\right)$.

*In the spatial case, investigated in this paper, case (D) can have two different outcomes, depending on the initial distribution of inoculants. We will refer to these as case (Da) and case (Db).) 


\section{Monostable traveling waves}

Cases (A) and (C): In these cases, the unstable steady state $\mathbf{U}_{w}^{*}$ will be invaded by a steady state that contains the commercial inoculant, where either coexistence of the inoculant with the resident community is observed $\left(\mathbf{U}_{c w}^{* *}\right.$, case $\left.(\mathrm{A})\right)$ or the resident community is displaced by the inoculant $\left(\mathbf{U}_{c}^{*}\right.$, case $(\mathrm{C})$ ). To find an estimate for the minimal propagation speed $c$, we linearize Eq. (9) around the unstable steady state $\mathbf{U}_{w}^{*}$ and obtain

$$
\left\{\begin{array}{l}
-c P^{\prime}=\left.F_{P}\right|_{\mathbf{U}_{w}^{*}}+\left.\frac{\partial F_{P}}{\partial P}\right|_{\mathbf{U}_{w}^{*}} P+\left.\frac{\partial F_{P}}{\partial M_{c}}\right|_{\mathbf{U}_{w}^{*}} M_{c}+\left.\frac{\partial F_{P}}{\partial M_{w j}}\right|_{\mathbf{U}_{w}^{*}} M_{w j} \\
-c M_{c}^{\prime}=D_{c} M_{c}^{\prime \prime}+\left.F_{M_{c}}\right|_{\mathbf{U}_{w}^{*}}+\left.\frac{\partial F_{M_{c}}}{\partial P}\right|_{\mathbf{U}_{w}^{*}} P+\left.\frac{\partial F_{M_{c}}}{\partial M_{c}}\right|_{\mathbf{U}_{w}^{*}} M_{c}+\left.\frac{\partial F_{M_{c}}}{\partial M_{w j}}\right|_{\mathbf{U}_{w}^{*}} M_{w j} \\
-c M_{w j}^{\prime}=D_{w} M_{w j}^{\prime \prime}+\left.F_{M_{w j}}\right|_{\mathbf{U}_{w}^{*}}+\left.\frac{\partial F_{M_{w j}}}{\partial P}\right|_{\mathbf{U}_{w}^{*}} P+\left.\frac{\partial F_{M_{w j}}}{\partial M_{c}}\right|_{\mathbf{U}_{w}^{*}} M_{c}+\left.\frac{\partial F_{M_{w j}}}{\partial M_{w j}}\right|_{\mathbf{U}_{w}^{*}} M_{w j}
\end{array}\right.
$$

Because $\left.F_{M_{c}}\right|_{\mathbf{U}_{w}^{*}}=0$ and $\left.\frac{\partial F_{M_{c}}}{\partial P}\right|_{\mathbf{U}_{w}^{*}}=\left.\frac{\partial F_{M_{c}}}{\partial M_{w j}}\right|_{\mathbf{U}_{w}^{*}}=0$, we can observe that the component $M_{c}$ of the linearized problem of Eq. (11) satisfies

$$
D_{c} M_{c}^{\prime \prime}+c M_{c}^{\prime}+F_{c}^{*} M_{c}=0,
$$

where

$$
F_{c}^{*}=P_{w}^{*}\left(\frac{q_{c m} \beta_{c} a_{w c}}{a_{w c}+N M_{w}^{*}}-\frac{q_{h m} \alpha_{c}}{P_{w}^{*}+d}\right) .
$$

From the analysis of the non spatial version of the model (Martignoni et al., 2020b), we know that $F_{c}^{*}>0$ for cases $(\mathrm{A})$ and $(\mathrm{C})$. Hence, one can conclude that a lower bound for the propagation speed $c_{c}^{*} \leq c$ is given by (Murray, 1993; Li et al., 2005)

$$
c_{c}^{*}=2 \sqrt{D_{c} F_{c}^{*}}=2 \sqrt{D_{c} P_{w}^{*}\left(\frac{q_{c m} \beta_{c} a_{w c}}{a_{w c}+N M_{w}^{*}}-\frac{q_{h m} \alpha_{c}}{P_{w}^{*}+d}\right)} .
$$

Thus in cases (A) and (C) the commercial inoculant invade a field inhabitated by a resident community at a minimal speed that increases with increasing dispersal ability of the commercial fungus and decreases with increasing diversity of the resident fungal community. Additionally, the minimal propagation speed increases if the commercial fungus can be regarded as a cheater, i.e. when $\alpha_{c}$ is small and $\beta_{c}$ is large. These findings are supported by numerical simulations. In Fig. S1, the theoretically predicted linear speed of Eq. (14) is compared with the speed resulting from numerical simulations, for different dispersal ability and mutualist quality of the inoculant, and for increasing diversity of the resident community. The speed resulting from the numerical simulations does not depend on whether the resident community is displaced (case (C)) or the inoculant coexist with the community (case (A)), as anticipated by the traveling wave analysis.

Case (B): In this case, the resident community competes strongly against the inoculant. The steady state $\mathbf{U}_{w}^{*}$ is stable while the unstable steady state is now $\mathbf{U}_{c}^{*}$. We linearize therefore Eq. (9) around $\mathbf{U}_{c}^{*}$. Because $\left.F_{M_{w j}}\right|_{\mathbf{U}_{c}^{*}}=0$ and $\left.\frac{\partial F_{M_{w j}}}{\partial P}\right|_{\mathbf{U}_{c}^{*}}=\left.\frac{\partial F_{M_{w j}}}{\partial M_{w j}}\right|_{\mathbf{U}_{c}^{*}}=0$, all components $M_{w j}$ of the linearized problem satisfy

$$
D_{w} M_{w}^{\prime \prime}+c M_{w}^{\prime}+F_{w}^{*} M_{w}=0
$$


where

$$
F_{w}^{*}=P_{c}^{*}\left(\frac{q_{c m} \beta_{w} a_{c w}}{a_{c w}+M_{c}^{*}}-\frac{q_{h m} \alpha_{w}}{P_{c}^{*}+d}\right)
$$

It is known that $F_{w}^{*}>0$ for case (B) (Martignoni et al., 2020b). A lower bound for the propagation speed $c_{w}^{*} \leq c$ is thus given by

$$
c_{w}^{*}=2 \sqrt{D_{w} F_{w}^{*}}=2 \sqrt{D_{w} P_{c}^{*}\left(\frac{q_{c m} \beta_{w} a_{c w}}{a_{c w}+M_{c}^{*}}-\frac{q_{h m} \alpha_{w}}{P_{c}^{*}+d}\right)} .
$$

One can conclude that in case (B) the commercial inoculant is displaced by the resident community at a minimal speed $c_{w}^{*}$. The speed increases with increasing mutualist quality of the inoculant (i.e., with larger $\alpha_{c} / \beta_{c}$ ratio, determining the value of $P_{c}^{*}$ and $M_{c}^{*}$ ) and with decreasing competitive strength of the inoculant $\left(a_{c w}\right)$, and it is independent on the dispersal ability of the inoculant. The linear speed of Eq. (17) seems to be a good approximation when the competitive ability of the inoculant is small (i.e., $a_{c w}$ is large, see Fig. S2c), and when the mutualist quality of the inoculant is low (i.e., the ratio $\alpha_{c} / \beta_{c}$ is small, see Fig. S2b). However the speed $c_{w}^{*}$ appears to be non-linear for higher competitive ability and mutualist quality of the inoculant.

\subsubsection{Bistable traveling wave}

Case (Da), when $N>1$ : In this case competition between the inoculant and the resident community is strong, and the steady state $\mathbf{U}_{w}^{*}$ and $\mathbf{U}_{c}^{*}$ are both stable. We will first discuss the case where $N>1$, i.e., the resident community consists of two or more fungal species. To understand which steady state will invade the other, we will determine the sign of the propagation speed $c$. For this purpose, we multiply Eq. (8b) by $M_{c}^{\prime}$ and integrate over the whole $z$-domain. We obtain the equation

$$
\int_{-\infty}^{\infty}\left[D_{c} M_{c}^{\prime \prime} M_{c}^{\prime}+c M_{c}^{\prime 2}+F_{M_{c}} M_{c}^{\prime}\right] \mathrm{d} z=0 .
$$

We know that $M_{c}^{\prime}=0$ when $z \rightarrow \pm \infty$. Hence $\int_{-\infty}^{\infty} M_{c}^{\prime \prime} M_{c}^{\prime} \mathrm{d} z=\int_{-\infty}^{\infty} 1 / 2\left(\left(M_{c}^{\prime}\right)^{2}\right)^{\prime} \mathrm{d} z=0$ and Eq. (18) can be rewritten as

$$
c \underbrace{\int_{-\infty}^{\infty}\left(M_{c}^{\prime}\right)^{2} \mathrm{~d} z}_{>0}=-\int_{-\infty}^{\infty} F_{M_{c}} M_{c}^{\prime} \mathrm{d} z .
$$

The sign of the wave speed $c$ depends therefore on the sign of the integral on the right-hand side of Eq. (19). We can observe that

$$
-\int_{-\infty}^{\infty} F_{M_{c}} M_{c}^{\prime} \mathrm{d} z=-\int_{-\infty}^{\infty} \frac{F_{M_{c}}}{M_{c}} M_{c}^{\prime} M_{c} \mathrm{~d} z=-\int_{-\infty}^{\infty} \frac{F_{M_{c}}}{2 M_{c}}\left(M_{c}^{2}\right)^{\prime} \mathrm{d} z .
$$

According to the boundary conditions of Eq. (10),

$$
\frac{F_{M_{c}}}{M_{c}}(-\infty)=\frac{F_{M_{c}}}{M_{c}}\left(\mathbf{U}_{c}^{*}\right)=0, \quad \text { and } \quad \frac{F_{M_{c}}}{M_{c}}(+\infty)=\frac{F_{M_{c}}}{M_{c}}\left(\mathbf{U}_{w}^{*}\right)=F_{c}^{*} .
$$

From Martignoni et al. (2020b) we know that, for case (D), $F_{c}^{*}<0$. Additionally, from numerical simulations one can notice that the slope of $M_{c}$ is really steep (see Table S2) and thus the integral of Eq. (20) can be approximated by

$$
-\int_{-\infty}^{\infty} \frac{F_{M_{c}}}{2 M_{c}}\left(M_{c}^{2}\right)^{\prime} \mathrm{d} z=\frac{1}{2}\left(\frac{F_{M_{c}}}{M_{c}}(\infty)-\frac{F_{M_{c}}}{M_{c}}(-\infty)\right)=\frac{1}{2} F_{c}^{*}<0 .
$$


Hence, the integral on the right side of Eq. (20) is always negative. We can conclude that the propagation speed $c$ is negative, and therefore the stable steady state $\mathbf{U}_{w}^{*}$ is expected to invade the state $\mathbf{U}_{c}^{*}$. In other words, the commercial inoculant is displaced by the resident fungal community.

To gain more insights into the speed at which the commercial inoculant is displaced, we explore the dependence of the speed on diversity of the resident community, and on the dispersal ability and mutualist quality of the inoculant numerically (see Fig. S3). We find that the speed at which the inoculant is displaced increases with increasing diversity in the resident community, and decreases with increased dispersal ability and with decreasing mutualist quality of the inoculant.

For a combination of low resident fungal density and high propagule pressure, the spatial dynamics follows a multi-step process illustrated in Fig. 1, case (Da), that is:

- The inoculant can temporarily establish and spread from its initial location. This initial spread prevents the growth of resident fungi in areas where the density of fungal inoculant is high, but at the same time plant growth is accelerated by the large presence of the inoculant (Fig. 1, case (Da), $\mathrm{T}=20$ ).

- Beyond the dispersal range of the inoculant, the density of resident fungi increases and so does plant productivity, eventually reaching a higher density than when only the inoculant is present (Fig. 1, case (Da), $\mathrm{T}=30$ and $\mathrm{T}=40$ ).

- A higher plant density determines higher resource availability, what gives a competitive advantage to the resident community with respect to the inoculant. The resident community is able to recolonize the portion of field taken by the inoculant leading the inoculated population to extinction (Fig. 1, case (Da), $\mathrm{T}=60$ and $\mathrm{T}=100$ ).

The speed at which the inoculant is displaced increases with increasing diversity of the resident fungal community, and decreases with increasing dispersal ability of the inoculant and with decreasing mutualist quality (see Fig. 2, case (Da)). For higher proportion of field occupied by the inoculant, resident fungi may not be able to recolonize the field (see Fig. 1, case (Db)).

Case (Da), when $N=1$ : We consider now the case where a strong competing inoculant $m_{c}$ is introduced in the field is inhabited by a single resident fungal species $m_{w}$, that is a strong competitor as well. In this case, the competitive exclusion steady states $\mathbf{U}_{w}^{*}$ (only $m_{w}$ ) and $\mathbf{U}_{c}^{*}$ (only $m_{c}$ ) are both stable. If $m_{c}$ and $m_{w}$ have different parameters, the strongest competitor will invade the other. A competitive advantage can be given by larger initial propagule density, lower mutualist quality (i.e., smaller $\alpha / \beta$ ratio) or lower maintenance cost (i.e., smaller $\mu_{m}$ ). A larger diffusion constant (i.e., larger $D_{c}$ or $D_{w}$ ), can drive the invasion of a species only if the domain was previoulsy unoccupied by the other species.

If the two fungi are identical, none of the species has a competitive advantage over the other and stationary solutions will be observed. To explore this scenario numerically, we consider on the left of the domain a steady state where $m_{w}$ is zero, and on the right a steady state a steady state where $m_{c}$ is zero. In this case, neither $m_{w}$ nor $m_{c}$ will prevail over the other. At the boundary between the two domains, where the two fungal species meet, we observe an increase in plant biomass density due to the partial presence of both species (see Fig. S4). The increase in plant biomass density is less pronounced for stronger competition between the fungi. 
Table S1: Description of variables and parameters of the model given in (6), with respective measurement units.

\begin{tabular}{|c|c|c|}
\hline Symbol & Description & Unit \\
\hline$p$ & Plant & plant biomass density \\
\hline$m_{c}$ & Fungal inoculant & fungal biomass density \\
\hline$m_{w}$ & resident fungal species & fungal biomass density \\
\hline$D_{c}$ & Diffusion coefficient of $m_{c}$ & $\frac{\text { space }}{\text { time }}$ \\
\hline$D_{w}$ & Diffusion coefficient of $m_{w}$ & \\
\hline$a_{w c}$ & Competition parameter $\left(m_{w}\right.$ toward $\left.m_{c}\right)$ & fungal biomass density \\
\hline$a_{c w}$ & Competition parameter $\left(m_{c}\right.$ toward $\left.m_{w}\right)$ & fungal biomass density \\
\hline$a_{w w}$ & Competition parameter $\left(m_{w}\right.$ toward $\left.m_{w}\right)$ & fungal biomass density \\
\hline$d$ & Half-saturation constant & plant biomass density \\
\hline$r_{p}$ & Intrinsic growth rate & $\frac{\text { phosphorus }}{\text { plant biomass density } \times \text { time }}$ \\
\hline$\alpha$ & Phosphorus exchange ability & $\frac{\text { phosphorus }}{(\text { time }) \times(\text { fungal biomass density) }}$ \\
\hline$\beta$ & Carbon exchange ability & $\frac{\text { carbon }}{(\text { time }) \times(\text { fungal biomass density }) \times(\text { plant biomass density }}$ \\
\hline$\mu_{p}$ & Plant maintenance rate & $\overline{(\text { time }) \times(\text { plant biomass density })}$ \\
\hline$\mu_{m}$ & Fungal maintenance rate & $\overline{(\text { time }) \times(\text { fungal biomass density })}$ \\
\hline$q_{h m}$ & $\begin{array}{l}\text { Conversion phosphorus to fungal biomass } \\
\text { density }\end{array}$ & fungal biomass density/ phosphorus \\
\hline$q_{h p}$ & $\begin{array}{l}\text { Conversion phosphorus to plant biomass } \\
\text { density }\end{array}$ & plant biomass density/ phosphorus \\
\hline$q_{c m}$ & $\begin{array}{l}\text { Conversion carbon to fungal biomass den- } \\
\text { sity }\end{array}$ & fungal biomass density/carbon \\
\hline$q_{c p}$ & $\begin{array}{l}\text { Conversion carbon to plant biomass den- } \\
\text { sity }\end{array}$ & plant biomass density/ carbon \\
\hline
\end{tabular}




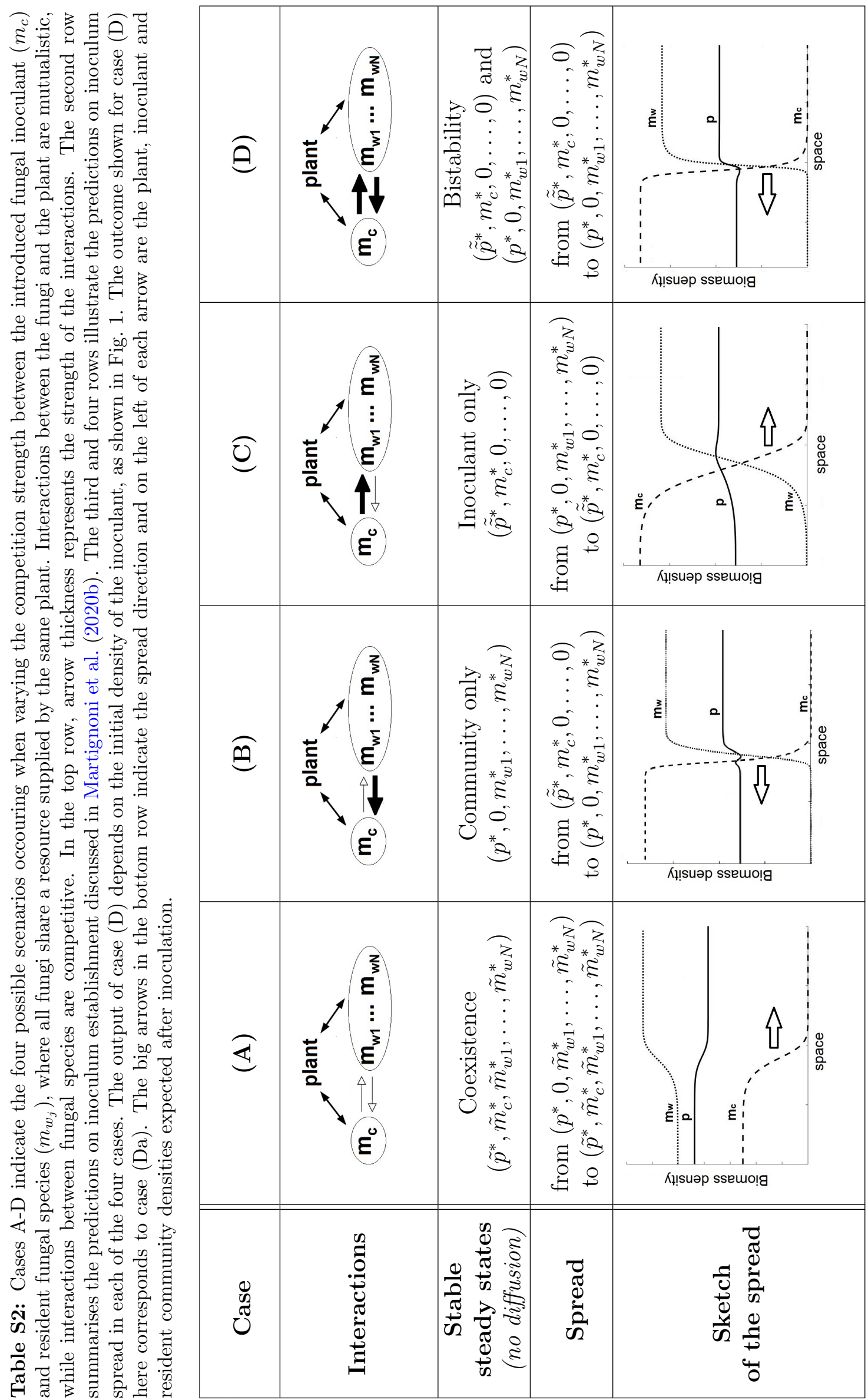


(a)

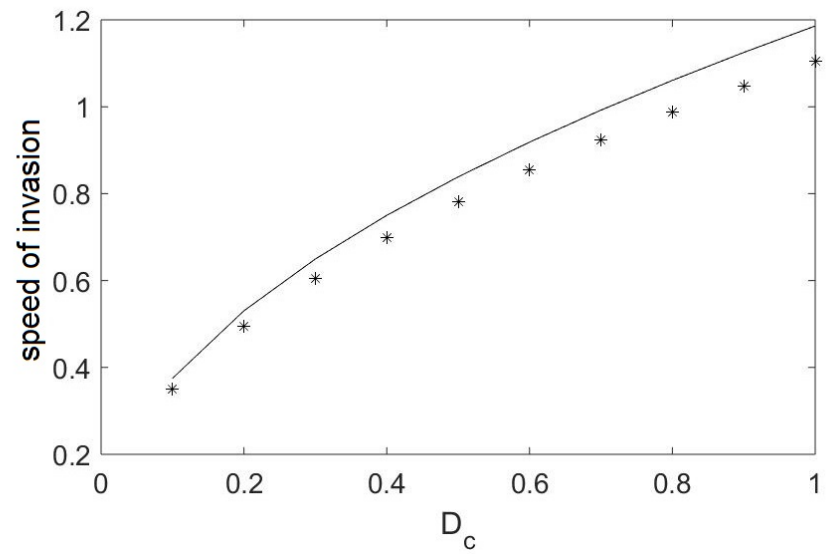

(b)

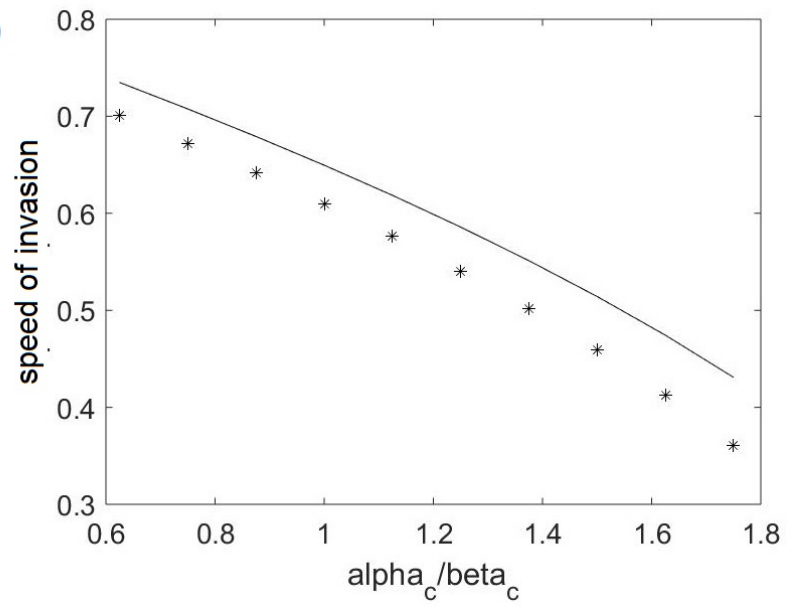

(c)

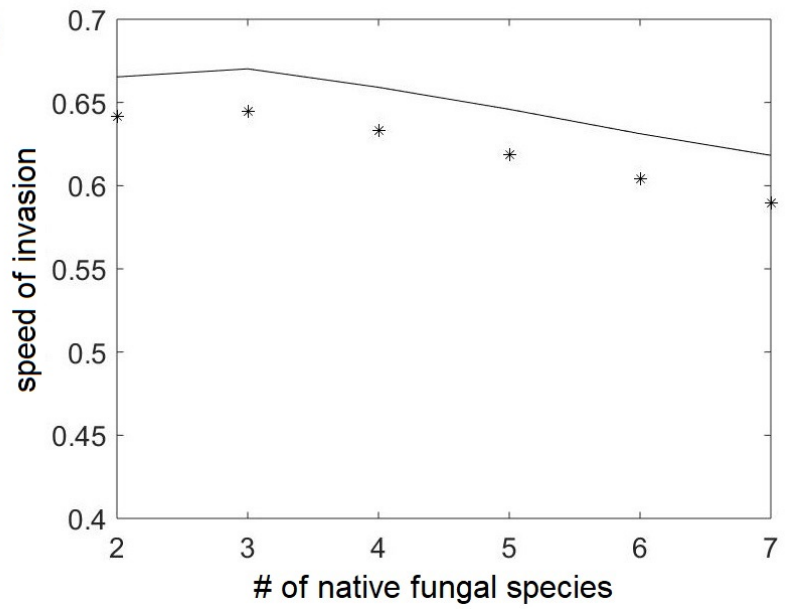

Fig. S1: Speed of invasion of the inoculant in a weak competing resident community (cases (A) and (C)) as a function of (a) the diffusion constant of the inoculant, (b) the mutualist quality of the inoculant, and (c) the number of species present in the resident community. The solid lines are the theoretically predicted lower bounds (see Eq. (14)) while the scattered plots are the results of the numerical simulations. The speed of invasion does not depend on whether the inoculant coexist with the resident community (case $(\mathrm{A})$ ) or displace the resident community (case (C)). In figures (a) and (b) competition parameters are $a_{w w}=a_{w c}=2.2, a_{c w}=0.3, \alpha_{w}=\beta_{w}=0.4$. In figure (c) $a_{w w}=a_{w c}=3.0, a_{c w}=0.1, \alpha_{w}=0.7$ and $\beta_{w}=0.3$. Other parameter values are $q_{h p}=3, q_{c m}=2$, $q_{h m}=q_{c p}=1, \mu_{p}=\mu_{m}=0.3, d=1.2, r_{p}=0.02, D_{w}=0.3$. 
(a)

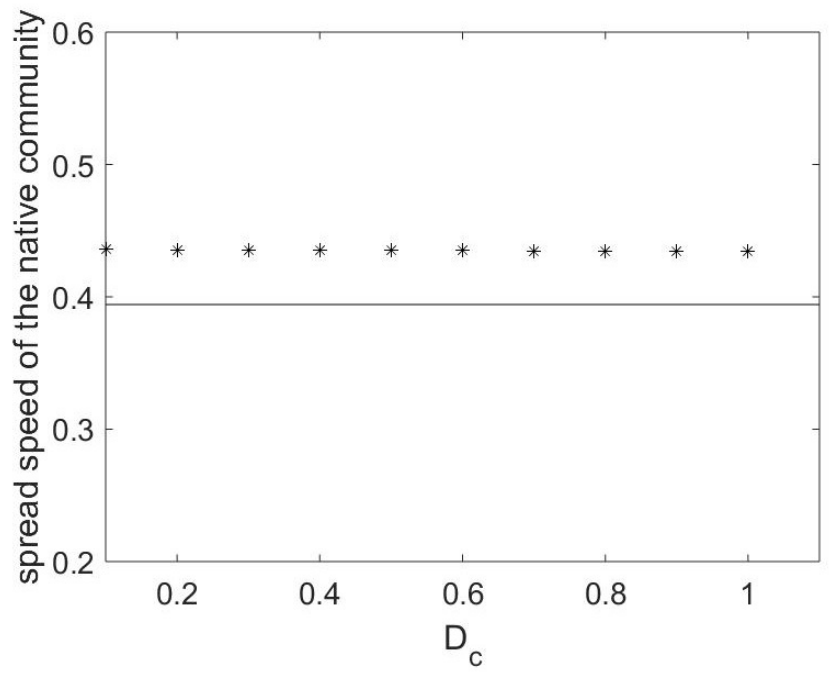

(b)

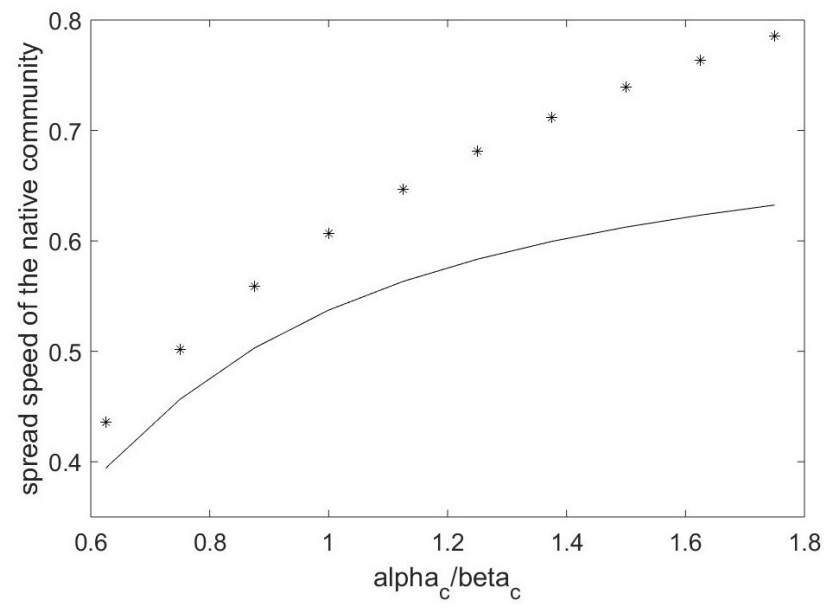

(c)

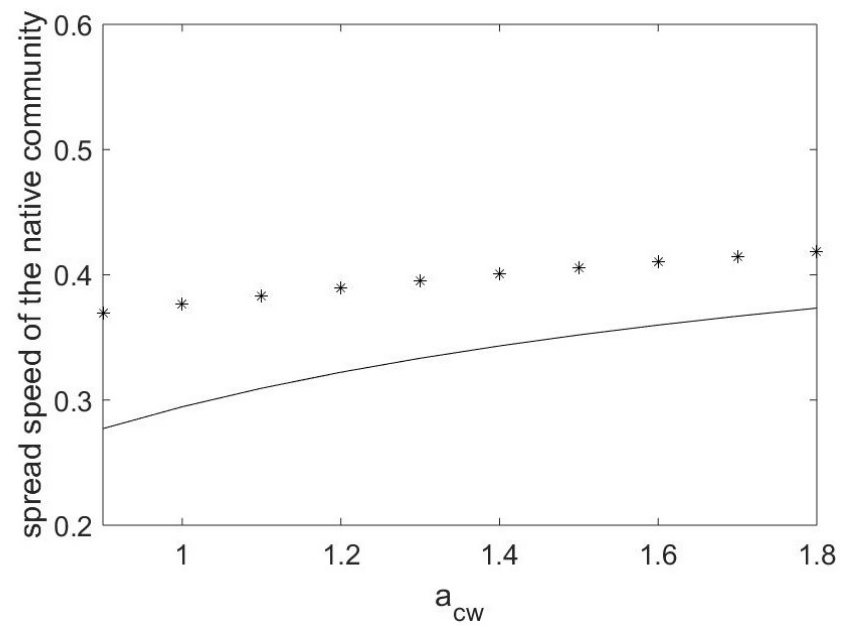

Fig. S2: Numerical simulations representing the speed at which a weakly competing inoculant is displaced by the resident community (case (B)). The solid lines are the theoretically predicted lower bounds (see Eq. (17)) while the scattered plots are the results of the numerical simulations. The speed is plot as a function of (a) the diffusion constant of the inoculant, (b) the mutualist quality of the inoculant, and (c) the competitive strength of the inoculant. Competition parameters are $a_{w w}=2.2$, $a_{w c}=0.3$. Other parameters correspond to those of Fig. S1a. 

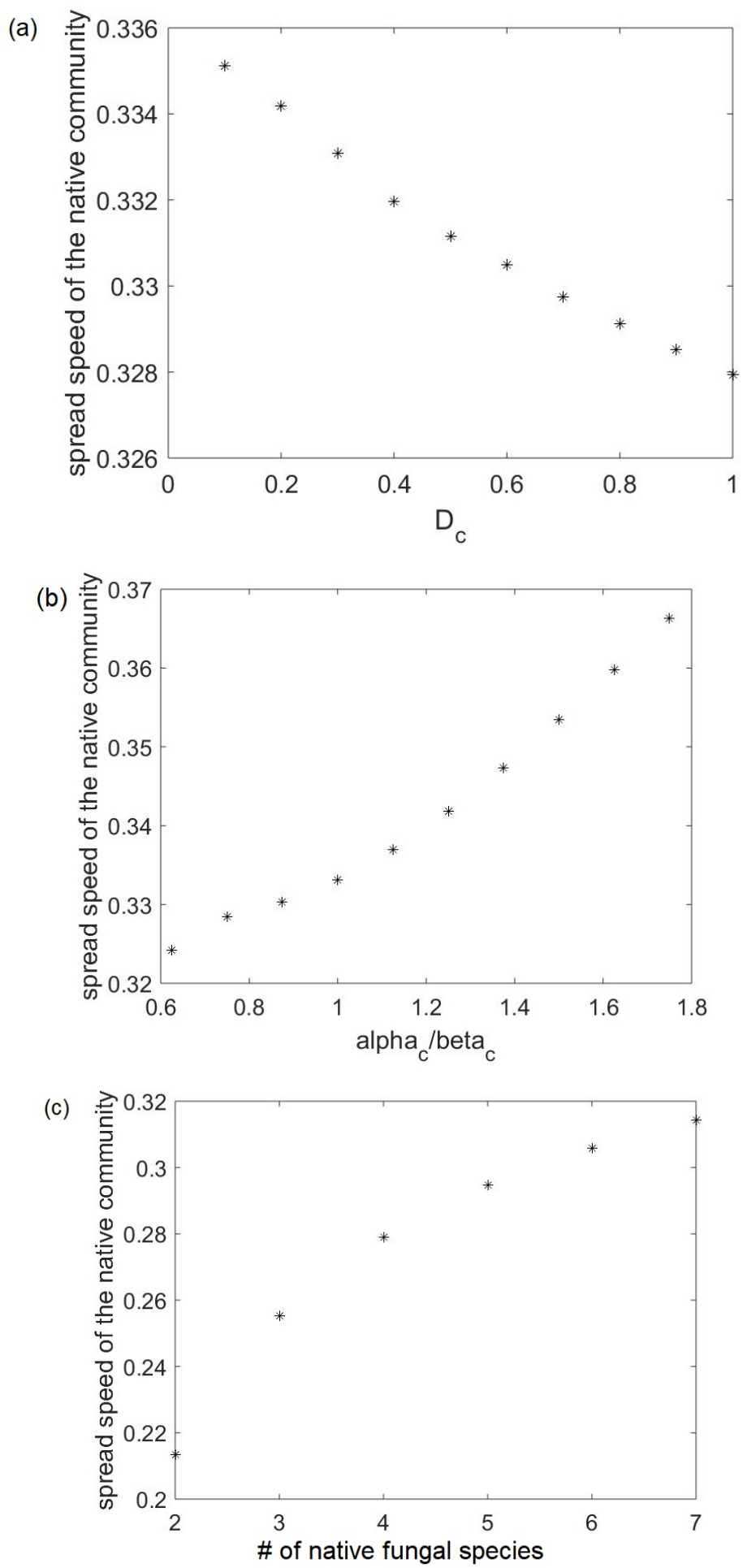

Fig. S3: Numerical simulations representing the speed at which a competitive inoculant is displaced by a strongly competing resident community (case (Da), for $N>1$ ), as a function of (a) the diffusion constant of the inoculant, (b) the mutualist quality of the inoculant, and (c) the number of species present in the resident community. Competition parameters are $a_{w w}=2.2, a_{w c}=a_{c w}=0.3$. Other parameters correspond to those of Fig. S1a. 


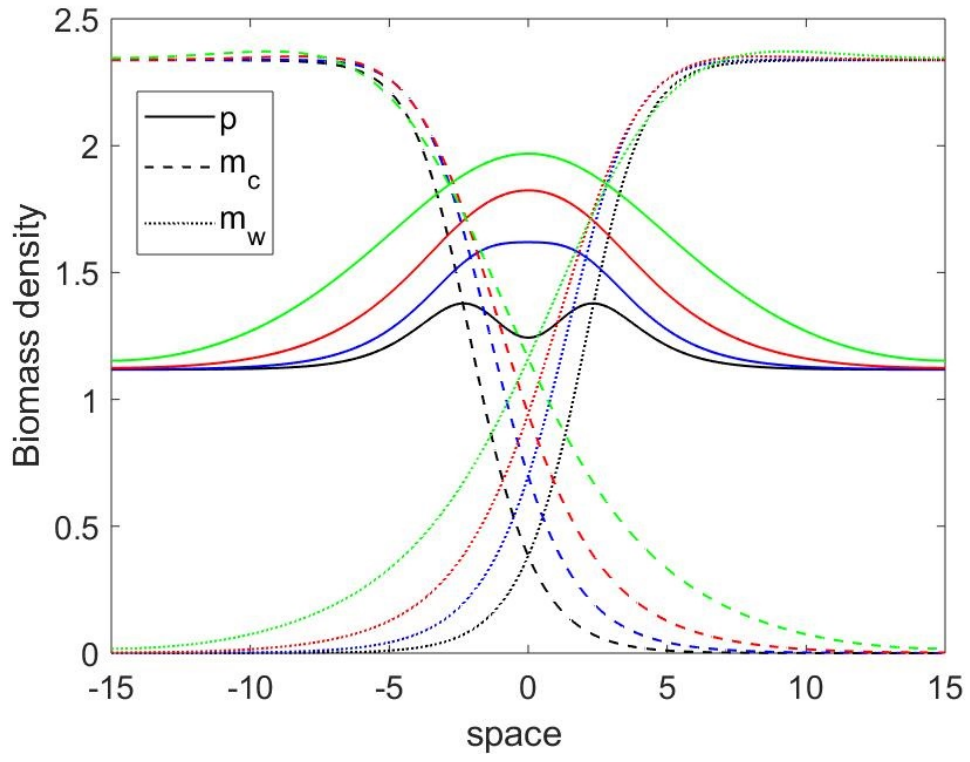

Fig. S4: Numerical simulation showing the plant (solid lines) and fungal biomass density distribution resulting from the encounter of two strongly competing fungi $\left(m_{c}\right.$, scattered line, and $m_{w}$, dotted line), for different competition strengths (case (D), for $N=1$ ). Competition parameters $a_{c w}$ and $a_{w c}$ have the same value and they are varies from 0.1 (strongest competition observed, black curves) to 0.7 (weakest competition observed, green curves) in steps of 0.2. Other parameters correspond to those of Fig. S1. 